\title{
HERSCHEL-ATLAS: A BINARY HyLIRG PINPOINTING A CLUSTER OF STARBURSTING PROTOELLIPTICALS
}

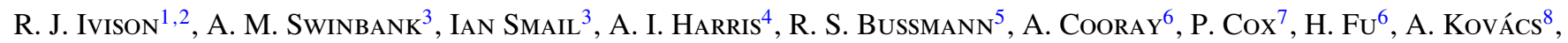
M. KRips ${ }^{7}$, D. NARAYANAN ${ }^{9}$, M. Negrello ${ }^{10}$, R. Neri ${ }^{7}$, J. Peñarrubia ${ }^{2}$, J. Richard ${ }^{11}$, D. A. Riechers ${ }^{12}$, K. Rowlands ${ }^{13}$, J. G. Staguhn ${ }^{14}$, T. A. TargetT ${ }^{2}$, S. Amber ${ }^{15}$, A. J. BakeR ${ }^{16}$, N. Bourne ${ }^{13}$, F. Bertoldi ${ }^{17}$, M. Bremer ${ }^{18}$, J. A. Calanog ${ }^{6}$, D. L. Clements ${ }^{19}$, H. Dannerbauer ${ }^{20}$, A. Dariush ${ }^{21}$, G. De Zotti ${ }^{10,22}$, L. Dunne ${ }^{23}$, S. A. Eales ${ }^{24}$, D. Farrah ${ }^{25}$, S. Fleuren ${ }^{26}$, A. Franceschini ${ }^{27}$, J. E. Geach ${ }^{28}$, R. D. GeOrGe ${ }^{2}$, J. C. Helly ${ }^{3}$, R. Hopwood ${ }^{15,19}$, E. Ibar ${ }^{1,29}$, M. J. JaRvis ${ }^{30,31}$, J.-P. KneiB ${ }^{32}$, S. Maddox ${ }^{23}$, A. Omont ${ }^{33}$, D. ScotT ${ }^{34}$, S. SerJeAnt ${ }^{15}$, M. W. L. Smith ${ }^{24}$, M. A. ThOMPSON ${ }^{28}$, E. VALIANTE ${ }^{24}$, I. VALTChanov ${ }^{35}$, J. VIEIRA ${ }^{36}$, AND P. VAN DER WerF ${ }^{37}$

${ }^{1}$ UK Astronomy Technology Centre, Science and Technology Facilities Council, Royal Observatory, Blackford Hill, Edinburgh EH9 3HJ, UK

${ }^{2}$ Institute for Astronomy, University of Edinburgh, Royal Observatory, Blackford Hill, Edinburgh EH9 3HJ, UK

${ }^{3}$ Institute for Computational Cosmology, Durham University, South Road, Durham DH1 3LE, UK

${ }^{4}$ Department of Astronomy, University of Maryland, College Park, MD 20742, USA

${ }^{5}$ Harvard-Smithsonian Center for Astrophysics, 60 Garden St, Cambridge, MA 02138, USA

${ }^{6}$ Department of Physics and Astronomy, University of California, Irvine, CA 92697, USA

${ }^{7}$ Institut de Radioastronomie Millimétrique, 300 rue de la Piscine, F-38406 Saint-Martin d'Hères, France

${ }^{8}$ Department of Astronomy, University of Minnesota, Minneapolis, MN 55414, USA

${ }^{9}$ Steward Observatory, University of Arizona, Tucson, AZ 85721, USA

${ }^{10}$ INAF, Osservatorio Astronomico di Padova, I-35122 Padova, Italy

${ }^{11}$ Centre de Recherche Astrophysique de Lyon, Université Lyon 1, 9 Avenue Charles André, F-69561 Saint Genis Laval Cedex, France

${ }^{12}$ Department of Astronomy, Space Science Building, Cornell University, Ithaca, NY 14853-6801, USA

${ }^{13}$ School of Physics and Astronomy, University of Nottingham, University Park, Nottingham NG7 2RD, UK

${ }^{14}$ The Henry A. Rowland Department of Physics and Astronomy, Johns Hopkins University, 3400 N. Charles Street, Baltimore, MD 21218, USA

${ }_{15}$ Department of Physical Sciences, The Open University, Milton Keynes, UK

${ }^{16}$ Department of Physics and Astronomy, Rutgers, The State University of New Jersey, Piscataway, NJ 08854-8019, USA

${ }^{17}$ Argelander-Institute for Astronomy, Bonn University, Auf dem Huegel 71, D-53121 Bonn, Germany

${ }^{18}$ H. H. Wills Physics Laboratory, University of Bristol, Tyndall Avenue, Bristol BS8 1TL, UK

${ }^{19}$ Physics Department, Imperial College London, South Kensington Campus, London SW7 2AZ, UK

${ }^{20}$ Institut für Astrophysik, Universität Wien, A-1180 Wien, Austria

${ }^{21}$ Institute of Astronomy, University of Cambridge, Madingley Road, Cambridge CB3 0HA, UK

${ }_{22}$ SISSA, via Bonomea 265, I-34136 Trieste, Italy

${ }^{23}$ Department of Physics and Astronomy, University of Canterbury, Private Bag 4800, Christchurch, New Zealand

${ }^{24}$ School of Physics and Astronomy, Cardiff University, Queen's Buildings, The Parade 5, Cardiff CF24 3AA, UK

${ }^{25}$ Department of Physics, Virginia Polytechnic Institute and State University, MC 0435, 910 Drillfield Drive, Blacksburg, VA 24061, USA

${ }^{26}$ School of Physics and Astronomy, Queen Mary University of London, Mile End Road, London E1 4NS, UK

${ }^{27}$ Dipartimento di Astronomia, Universita' di Padova, I-35122 Padova, Italy

${ }^{28}$ Centre for Astrophysics Research, University of Hertfordshire, Hatfield AL10 9AB, UK

${ }^{29}$ Departamento de Astronomía y Astrofísica, Universidad Católica de Chile, Vicuña Mackenna 4860, Casilla 306, Santiago 22, Chile

${ }^{30}$ Astrophysics, Department of Physics, University of Oxford, Keble Road, Oxford OX1 3RH, UK

${ }^{31}$ Department of Physics, University of the Western Cape, South Africa

32 EPFL SB IPEP LASTRO, Observatoire Sauverny, CH-1290 Versoix, Switzerland

${ }^{33}$ UPMC Univ. Paris 06 and CNRS UMR7095 IAP, F-75014 Paris, France

${ }^{34}$ Department of Physics and Astronomy, University of British Columbia, Vancouver V6T 1Z1, Canada

${ }^{35}$ European Space Astronomy Centre, Herschel Science Centre, ESA, E-28691 Villanueva de la Cañada, Spain

${ }^{36}$ California Institute of Technology, 1200 East California Boulevard, Pasadena, CA 91125, USA

${ }^{37}$ Leiden Observatory, Leiden University, P.O. Box 9513, NL-2300 RA Leiden, The Netherlands Received 2013 February 12; accepted 2013 June 11; published 2013 July 17

\begin{abstract}
Panchromatic observations of the best candidate hyperluminous infrared galaxies from the widest Herschel extragalactic imaging survey have led to the discovery of at least four intrinsically luminous $z=2.41$ galaxies across an $\approx 100 \mathrm{kpc}$ region-a cluster of starbursting protoellipticals. Via subarcsecond interferometric imaging we have measured accurate gas and star formation surface densities. The two brightest galaxies span $\sim 3 \mathrm{kpc}$ FWHM in submillimeter/radio continuum and CO $J=4-3$, and double that in CO $J=1-0$. The broad CO line is due partly to the multitude of constituent galaxies and partly to large rotational velocities in two counter-rotating gas disks - a scenario predicted to lead to the most intense starbursts, which will therefore come in pairs. The disks have $M_{\text {dyn }}$ of several $\times 10^{11} M_{\odot}$, and gas fractions of $\sim 40 \%$. Velocity dispersions are modest so the disks are unstable, potentially on scales commensurate with their radii: these galaxies are undergoing extreme bursts of star formation, not confined to their nuclei, at close to the Eddington limit. Their specific star formation rates place them $\gtrsim 5 \times$ above the main sequence, which supposedly comprises large gas disks like these. Their high star formation efficiencies are difficult to reconcile with a simple volumetric star formation law. $N$-body and dark matter simulations suggest that this system is the progenitor of a $\mathrm{B}$ (inary)-type $\approx 10^{14.6}-M_{\odot}$ cluster.
\end{abstract}

Key words: galaxies: high-redshift - galaxies: starburst - infrared: galaxies - radio continuum: galaxies - radio lines: galaxies - submillimeter: galaxies

Online-only material: color figures 


\section{INTRODUCTION}

Of the known denizens of the galaxy zoo, hyperluminous infrared (IR) galaxies (HyLIRGs; $L_{\mathrm{IR}} \geqslant 10^{13} L_{\odot}$, where the IR luminosity is measured across $\lambda_{\text {rest }}=8-1000 \mu \mathrm{m}$ ) are among the rarest and most extreme. They provide excellent laboratories with which to confront the most recent hydrodynamic simulations of isolated and merging galaxies (e.g., Hayward et al. 2011). The $L_{\mathrm{IR}}$ of an HyLIRG implies a staggering star formation rate, SFR $\gtrsim 1000 M_{\odot} \mathrm{yr}^{-1}$ (Kennicutt 1998, for a Chabrier 2003 initial mass function (IMF)), unless the IMF is top-heavy, or there is a substantial contribution to $L_{\mathrm{IR}}$ from a deeply obscured active galactic nucleus (AGN), in which case we are seeing intense star formation accompanied by the rapid growth of a massive black hole (Alexander et al. 2008). Either way, we are witnessing galaxy formation at its most extreme.

How best can we identify the most luminous star-forming HyLIRGs? A promising method involves searching among the brightest sources detected in the widest surveys with Herschel. These comprise blazars, low-redshift spirals, and some intrinsically fainter sources that have been strongly lensed (e.g., Negrello et al. 2010). However, there is also the intriguing possibility that such samples may contain unlensed and thus intrinsically luminous galaxies - the rarest HyLIRGs are predicted to have a space density, $\approx 10^{-7} \mathrm{Mpc}^{-3}$ and may mark the sites of today's clusters (Negrello et al. 2005; Lapi et al. 2011).

Of the facilities providing redshifts for bright Herschel sources, the $100 \mathrm{~m}$ Robert C. Byrd Green Bank Telescope (GBT), together with the ultrawide-bandwidth Zpectrometer cross-correlation spectrometer and $K a$-band receiver, have been among the most effective (e.g., Swinbank et al. 2010; Frayer et al. 2011). Having added considerably to the sample of such sources with known redshifts, Harris et al. (2012) identified a flat trend of $L_{\mathrm{CO}}^{\prime}$ with ${ }^{12} \mathrm{C}^{16} \mathrm{O} J=1-0$ line $^{38}$ width. This contrasted with the steep power-law relation between $L_{\mathrm{CO}}^{\prime}$ and CO line width found by Bothwell et al. (2013) for unlensed submillimeter galaxies (SMGs), as expected if the most gasrich galaxies tend to live in the most massive gravitational potentials. Harris et al. argued that intrinsically fainter sources require a higher lensing magnification, $\mu$, to rise above the observational detection threshold for $\mathrm{CO} J=1-0$, which is approximately constant in flux. The flat trend seen in the GBT sample is then best interpreted as lensing magnification acting on a population with intrinsically steep number counts. Those GBT sources closest to the power-law fit seen for SMGs - typically those with the widest lines-are likely to be suffering the least lensing magnification. This is where we might expect to find any HyLIRGs that are lurking among the lensed starbursts.

Our approach here, therefore, is to search for HyLIRGs among those bright Herschel lens candidates with the broadest CO lines, starting with the best example in the sample observed with GBT by Harris et al., HATLAS J084933.4+021443 (hereafter HATLAS J084933, with $350 \mu \mathrm{m}$ flux density, $S_{350}=$ $293 \mathrm{mJy}$ ), which displays a line consistent with CO $J=1-0$ at $z_{\mathrm{LSR}}=2.410 \pm 0.003$, with an FWHM of $1180 \pm 320 \mathrm{~km} \mathrm{~s}^{-1}$ and $S_{\mathrm{CO} 1-0}=0.83 \pm 0.19 \mathrm{mJy}$. The amplification predicted by Harris et al. for HATLAS J084933 is consistent with unity, $\mu=2 \pm 1$.

\footnotetext{
${ }^{38}$ Hereafter, $\mathrm{CO}$ refers to ${ }^{12} \mathrm{C}^{16} \mathrm{O}$ unless stated otherwise.
}

In the next section, we describe an extensive set of observations. We present, analyze, interpret, and discuss our reduced images, spectra, and cubes in Section 3, finishing with our conclusions in Section 4. We adopt a cosmology with $H_{0}=71 \mathrm{~km} \mathrm{~s}^{-1} \mathrm{Mpc}^{-1}, \Omega_{\mathrm{m}}=0.27$, and $\Omega_{\Lambda}=0.73$, so $1^{\prime \prime}$ equates to $8.25 \mathrm{kpc}$ at $z=2.41$.

\section{OBSERVATIONS AND DATA REDUCTION}

Herschel imaging, undertaken as part of the wide-field $H$-ATLAS imaging survey (Eales et al. 2010), led to the selection of HATLAS J084933 as a potentially distant, lensed starburst: distant, because it is an example of a so-called $350 \mu \mathrm{m}$ peaker, with its thermal dust peak between the 250 and $500 \mu \mathrm{m}$ bands; lensed, because models of the far-IR/submillimeter (submm) source counts suggest that the majority of objects with $S_{500}>100 \mathrm{mJy}$ are expected to be either local $(z<0.1)$ or lensed (e.g., Negrello et al. 2010), along with the occasional flat-spectrum radio quasar.

On the basis that it represented the best chance of finding an intrinsically luminous system rather than a lensed galaxy, following the arguments laid out in Section 1, HATLAS J084933 was selected for observations with the Jansky Very Large Array (JVLA). Here, we detail the JVLA observations and those obtained with other facilities that followed as a consequence of our initial findings, presenting these data sets in order of decreasing wavelength.

\subsection{JVLA CO J $=1-0$ and $5 \mathrm{GHz}$ Continuum Imaging}

While the National Radio Astronomy Observatory's (NRAO's) $\mathrm{JVLA}^{39}$ was in its DnC, C, B, BnA, and A configurations, between 2012 January and 2013 January, we acquired $\approx 30 \mathrm{hr}$ of $\mathrm{Ka}$-band data, scheduled dynamically to ensure excellent atmospheric phase and pointing stability. We recorded two sets of eight contiguous baseband pairs, $1024 \times 2 \mathrm{MHz}$ dual-polarization channels in total. We tuned the first set of basebands to cover ${ }^{13} \mathrm{C}^{16} \mathrm{O}$ and ${ }^{12} \mathrm{C}^{18} \mathrm{O} J=1-0$. The ${ }^{12} \mathrm{C}^{16} \mathrm{O}$ $J=1-0$ transition $\left(v_{\text {rest }}=115.271203 \mathrm{GHz}\right.$; Morton \& Noreau 1994) was placed in the second set of baseband pairs, offseting down by $64 \mathrm{MHz}$ to $33.740 \mathrm{GHz}$ to avoid baseband edges.

Around $4 \mathrm{hr}$ of A-configuration $C$-band data were also obtained, recording $1024 \times 2 \mathrm{MHz}$ dual-polarization channels every $1 \mathrm{~s}$ across $4.2-6.5 \mathrm{GHz}$, with a small gap. Typically, $0.3 \mathrm{GHz}$ was lost to severe radio-frequency interference near $6.1 \mathrm{GHz}$, yielding a band center of $5.1 \mathrm{GHz}(5.9 \mathrm{~cm})$.

PKS J0825+0309 and PKS J0839+0319 were observed every few minutes to determine accurate complex gain solutions and bandpass corrections in the $K a$ and $C$ bands, respectively. 3C 286 was observed to set the absolute flux density scale, and the pointing accuracy was checked locally every hour.

The data were reduced using AIPS. The basebands were knitted together using the NOIFS and VBGLU tasks, yielding $u v$ data sets with two intermediate frequencies, each comprising $512 \times 2 \mathrm{MHz}$ channels.

For the spectral-line data, these were then imaged in groups of four channels $\left(71 \mathrm{~km} \mathrm{~s}^{-1}\right)$, with natural weighting. A variety of Gaussian tapers were used to weight the data, with distances to

\footnotetext{
39 This work is based on observations carried out with the JVLA. The NRAO is a facility of the NSF operated under cooperative agreement by Associated Universities, Inc.
} 


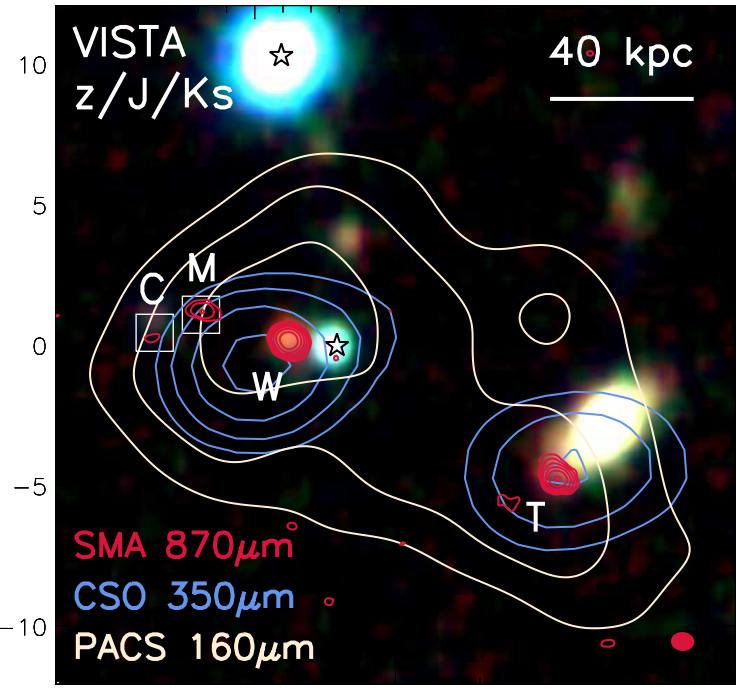

10 5 0 $-5$

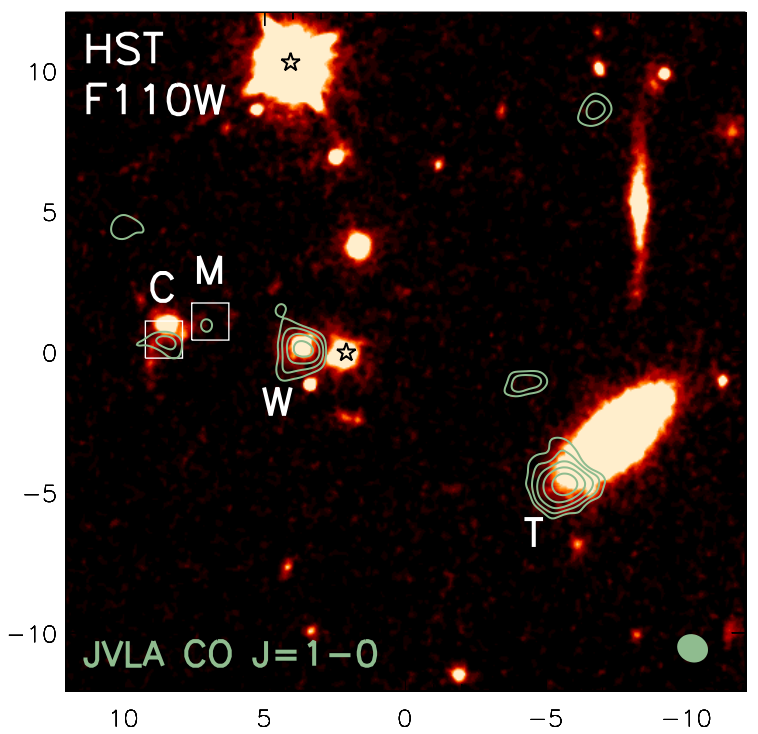

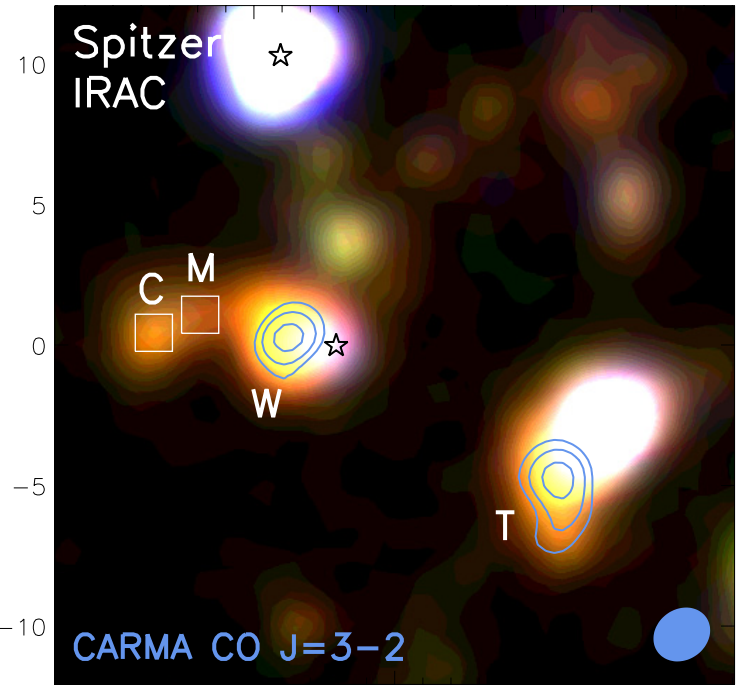

10
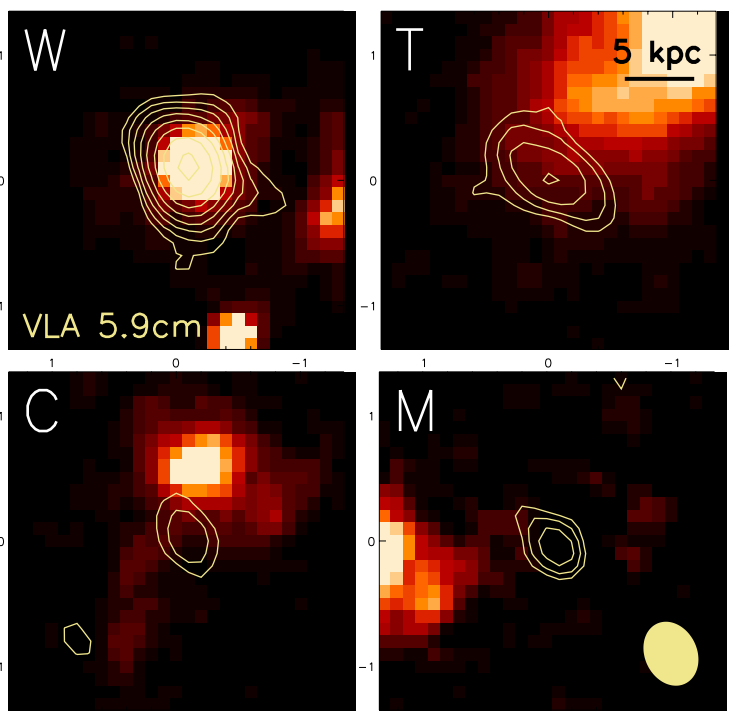

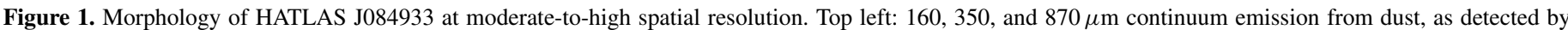

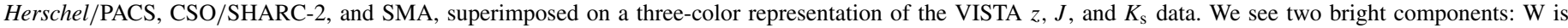

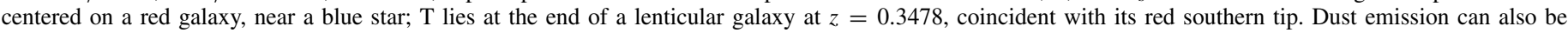

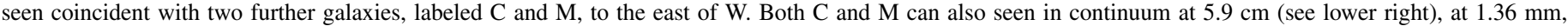

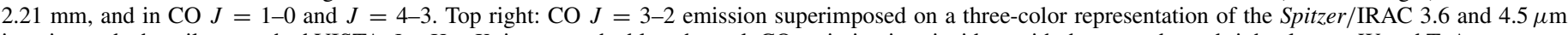

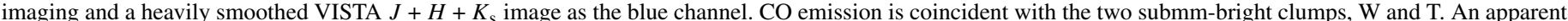

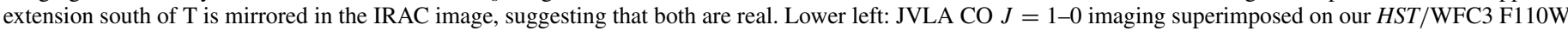

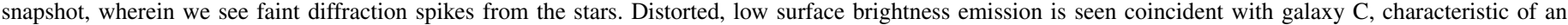

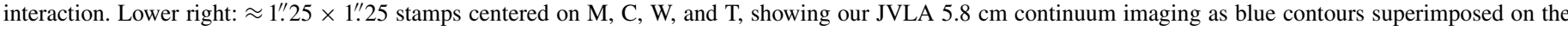

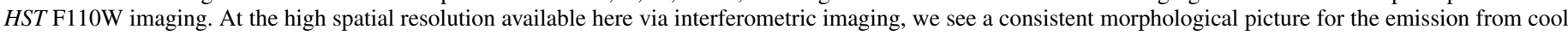

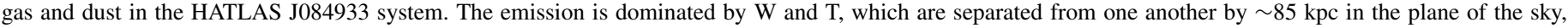

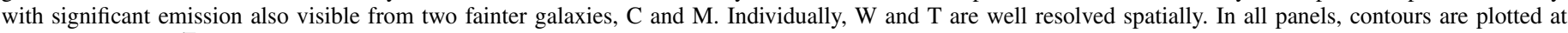

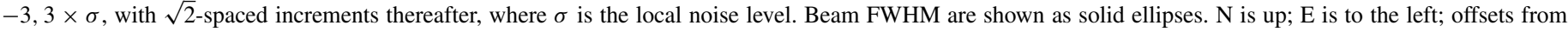
$\alpha_{2000}=132.3889$ and $\delta_{2000}=2.2457$ are marked in arcseconds. Known stars are labeled.

(A color version of this figure is available in the online journal.)

the $30 \%$ point of the Gaussian ranging from 100 to $1000 \mathrm{k} \lambda$, to form a number of cubes with FWHM spatial resolutions ranging from $00^{\prime} .53 \times 0$ "'.51 to 2 ". $0 \times 11^{\prime \prime} 8$. The line-integrated map shown in Figure 1 has a 1". $1 \times 1$ 1. 0 synthesized beam.

The naturally weighted $5.1 \mathrm{GHz}$ pseudocontinuum data were imaged, averaging all of the channels, loosely following the techniques outlined by Owen \& Morrison (2008). The resulting map has a spatial resolution of $0^{\prime} .53 \times 00^{\prime} .42$ (P.A., $26^{\circ}$ ) and an rms noise level of $2.9 \mu \mathrm{Jy}$ beam $^{-1}$ (Figure 1).

\section{2. $C O J=3-2$ Imaging from CARMA}

We used CARMA $^{40}$ to observe the CO $J=3-2$ line $\left(v_{\text {rest }}=345.795991 \mathrm{GHz}\right.$, redshifted to $\left.v_{\mathrm{obs}}=101.406 \mathrm{GHz}\right)$.

\footnotetext{
40 CARMA construction was derived from the states of Maryland, California and Illinois, the James S. McDonnell Foundation, the Gordon and Betty Moore Foundation, the Kenneth T. and Eileen L. Norris Foundation, the University of Chicago, the Associates of the California Institute of Technology, and the NSF Ongoing CARMA development and operations are supported by the NSF under a cooperative agreement, and by the CARMA partner universities.
} 
Observations were carried out with the $3 \mathrm{~mm}$ receivers and the CARMA spectral-line correlator, with an effective bandwidth of $3.7 \mathrm{GHz}$ per sideband and a spectral resolution of $15 \mathrm{~km} \mathrm{~s}^{-1}$. Four tracks were obtained between 2012 February 13 and 17 in the C configuration (15-352 m baselines), with 14 usable antennas and a total on-source observing time of $13.5 \mathrm{hr}$. The nearby quasar, PKS J0757+0956, was used for complex gain calibration. The bandpass shape and absolute flux calibration (the latter good to $\sim 15 \%$ ) were derived from observations of Mars, PKS J0854+2006 and 3C 84. Using natural weighting, we obtained an FWHM beam size of $2^{\prime \prime} .1 \times 1^{\prime \prime} .8$ (P.A., $126^{\circ}$ ), with a noise level, $\sigma=0.3 \mathrm{mJy}^{\text {beam }}{ }^{-1}$ (Figure 1 ).

\section{3. $C O \mathrm{~J}=4-3$ Imaging from IRAM PdBI}

During 2012 February, we obtained data using six $15 \mathrm{~m}$ antennas in the most extended configuration of the Institut de Radioastronomie Millimétrique's Plateau de Bure Interferometer (IRAM ${ }^{41} \mathrm{PdBI}$ ), with baselines ranging up to $800 \mathrm{~m}$.

The observing frequency was set to $135.203 \mathrm{GHz}$, the redshifted frequency of the CO $J=4-3$ line $\left(v_{\text {rest }}=\right.$ $461.04077 \mathrm{GHz}$ ). The weather conditions were exceptionally good with a precipitable water vapor of around $2 \mathrm{~mm}$ and $T_{\text {sys }} \sim$ $100 \mathrm{~K}$ on average. PKS J0825+0309 and PKS J0909+0121 were used to calibrate the complex gains, while 3C 84 and MWC 349 were chosen as bandpass and absolute flux calibrators. The rms noise level is $0.97 \mathrm{mJy}_{\text {beam }}{ }^{-1}$ in $4 \mathrm{MHz}$ wide spectral channels, for a synthesized beam measuring 1".0 0 0.'5 FWHM (P.A., $24^{\circ}$ ). Using only the line-free channels, the rms noise level is $47 \mu \mathrm{Jy}_{\text {beam }^{-1}}$.

We also report continuum photometry at $1.36 \mathrm{~mm}$ from observations obtained throughout 2012 as part of a search for water that will be reported elsewhere.

\section{4. $870 \mu m$ Continuum Imaging from the SMA}

Approximately 1.4, 4.9, and $1.7 \mathrm{hr}$ of integration were obtained using the Submillimeter Array $\left(\mathrm{SMA}^{42}\right)$ in its compact, extended, and very extended array configurations during 2011 December 8, 2012 January 31, and 2012 March 31, respectively (2011B-S044). The receivers were tuned such that the upper sideband was centered at $345 \mathrm{GHz}(870 \mu \mathrm{m})$, with $8 \mathrm{GHz}$ of single-polarization bandwidth in total. 3C 84 was used as the bandpass calibrator and Titan was used for absolute flux calibration. PKS J0825+0309 and PKS J0909+0121 were again used to track and check the phase and gain.

A ROBUST $=2$ weighting scheme resulted in a $0^{\prime \prime} 83 \times$ 0.67 beam FWHM (P.A., 86 ${ }^{\circ}$ ) and a noise level, $\sigma=$ $0.77 \mathrm{mJy}^{\text {beam }}{ }^{-1}$ (Figure 1).

\section{5. $350 \mu m$ Continuum Imaging with CSO/SHARC-2}

A total of 80 minutes of data were collected at $350 \mu \mathrm{m}$ during periods of excellent weather between 2012 January 12 and 13 at the $10.4 \mathrm{~m}$ Caltech Submillimeter Observatory $\left(\mathrm{CSO}^{43}\right)$, using the SHARC-2 camera (Dowell et al. 2003). We took advantage of the open-loop, actuated surface of the telescope (Leong

\footnotetext{
41 IRAM is supported by INSU/CNRS (France), MPG (Germany), and IGN (Spain).

${ }^{42}$ The SMA is a joint project between the Smithsonian Astrophysical Observatory and the Academia Sinica Institute of Astronomy and

Astrophysics, and is funded by the Smithsonian Institution and the Academia Sinica.

43 This material is based upon work at the CSO, which is operated by the California Institute of Technology under cooperative agreement with the National Science Foundation (AST-0838261).
}

et al. 2006) and we checked and updated the focus settings several times each night, resulting in near-Gaussian, diffractionlimited (8".5 FWHM) beam profiles. Data were reduced using CRUSH (Kovács 2008) and the resulting maps have a noise level, $\sigma=8 \mathrm{mJy} \mathrm{beam}^{-1}$ (Figure 1).

\subsection{Continuum Imaging from Herschel}

The acquisition and reduction of Herschel (Pilbratt et al. 2010) parallel-mode data from SPIRE (Griffin et al. 2010) and PACS (Poglitsch et al. 2010) for the $9 \mathrm{hr}$ Science Demonstration Phase field of $H$-ATLAS (Eales et al. 2010) are described in detail by Ibar et al. (2010a), Pascale et al. (2011), and Rigby et al. (2011). Here, we use the original SPIRE imaging and have obtained much deeper PACS data from the оT1 program, OT1_RIVISON_1. We recorded data simultaneously at 100 and $160 \mu \mathrm{m}$ while tracking across the target at $20^{\prime \prime} \mathrm{s}^{-1}$ in $103^{\prime}$ strips, each offset orthogonally by $4^{\prime \prime}$. A total of $180 \mathrm{~s}$ on source was acquired for each of two near-orthogonal scans. These data were tackled with a variant of the pipeline developed by Ibar et al. (2010b), reaching $\sigma \approx 4$ and $7 \mathrm{mJy}$ at 100 and $160 \mu \mathrm{m}$, respectively. The $160 \mu \mathrm{m}$ PACS image is shown in Figure 1.

\subsection{Infrared Continuum Imaging from Spitzer, VISTA, and the Hubble Space Telescope}

The 3.6 and $4.5 \mu \mathrm{m}$ images were acquired using the Infrared Array Camera (IRAC; Fazio et al. 2004) on board Spitzer ${ }^{44}$ (Werner et al. 2004) on 2012 June 24 as part of program 80156. The imaging involved a 36 position dither pattern, with a total exposure time of just over $1 \mathrm{ks}$, reaching rms depths of 0.3 and $0.4 \mu \mathrm{Jy}$ at 3.6 and $4.5 \mu \mathrm{m}$, respectively. Corrected basic calibrated data, pre-processed by the Spitzer Science Center, were spatially aligned and combined into mosaics (shown in Figure 1) with a re-sampled pixel size of 0.6 and angular resolution of $2^{\prime \prime}-2$.'5, using version 18.5.0 of MOPEX (Makovoz \& Marleau 2005).

Images were obtained in $Z, Y, J, H$, and $K_{\mathrm{s}}$ as part of VIKING, a public survey with the $4 \mathrm{~m}$ Visible and Infrared Survey Telescope for Astronomy (VISTA) at Paranal, Chile (Emerson \& Sutherland 2010), with an image quality of 0.9 FWHM, as shown in Figure 1.

A SNAPshot observation was obtained with the Hubble Space Telescope $^{45}$ (HST) on 2012 February 8, as part of Cycle-19 proposal 12488, using Wide-Field Camera 3 (WFC3) with its wide $J$ filter, F110W. The total exposure time was 252 s. Data were reduced using the IRAF MultiDrizzle package. Individual frames were corrected for distortion, cleaned by cosmic rays and other artifacts and median combined. The resulting $\sim 2^{\prime} \times 2^{\prime}$ image was re-sampled to a finer pixel scale of $0^{\prime \prime} .064$ and is shown in Figure 1.

\subsection{Optical Spectroscopy from Keck}

We observed several optical/IR sources in the vicinity of HATLAS J084933 using the DEIMOS spectrograph on the $10 \mathrm{~m}$

\footnotetext{
44 This work is based in part on observations made with the Spitzer Space Telescope, which is operated by the Jet Propulsion Laboratory, California Institute of Technology under a contract with NASA.

45 Based on observations made with the NASA/ESA Hubble Space Telescope, obtained at the Space Telescope Science Institute, which is operated by the Association of Universities for Research in Astronomy, Inc., under NASA contract NAS 5-26555. These observations are associated with program 12488.
} 
Keck II telescope ${ }^{46}$ during 2012 March 2. DEIMOS was used with its 600ZD grating (ruled with 600 lines $\mathrm{mm}^{-1}$ ), the GG455 filter, and a $1^{\prime \prime}$ slit at a central wavelength of $720 \mathrm{~nm}$. Two positions were targeted, each with a different position angle (P.A.), for 40 and 75 minutes.

\section{RESULTS, ANALYSIS, AND DISCUSSION}

\subsection{Basic Morphological Description}

At the spatial resolution of SPIRE, HATLAS J08493 is essentially unresolved. However, with only modestly improved spatial resolution, via deep imaging with PACS at 100-160 $\mu \mathrm{m}$ and CSO/SHARC-2 at $350 \mu \mathrm{m}$, the system is resolved into two sources, referred to hereafter as $\mathrm{W}$ and $\mathrm{T}$ (Figure 1).

Moving to higher spatial resolution, via interferometric imaging with SMA, IRAM PdBI, and CARMA at rest frame 255-880 $\mu \mathrm{m}$, we see a consistent morphological picture for the emission from cool dust in the HATLAS J084933 system (Figure 1). The dust continuum emission is dominated by $\mathrm{W}$ and $\mathrm{T}$, which are separated by $10^{\prime \prime} .5$, or $\sim 85 \mathrm{kpc}$ in the plane of the sky, with $\mathrm{W}$ being the brightest of the two from the optical all the way through to the radio regime. $\mathrm{W}$ is among the brightest unlensed SMGs ever observed interferometrically at submm wavelengths, with $\mathrm{T}$ being not far behind (Gear et al. 2000; Lutz et al. 2001; Younger et al. 2007, 2008, 2009; Wang et al. 2007; Aravena et al. 2010; Smolčić et al. 2012a, 2012b). Among the unlensed SMG population, its flux density has been equaled only by SMM J123711+622212 (also known as GN 20, $S_{870}=22.9 \pm 2.8$-Pope et al. 2005; Iono et al. 2006).

Aided by our interferometric astrometry we can see that at optical/IR wavelengths the westernmost clump, T, is barely visible as a red extension to a lenticular galaxy (Figure 1). W coincides with the extremely red component of a red/blue pair, separated by $\approx 2^{\prime \prime}$. Based on experience with SMGs, such as SMM J02399-0136 (e.g., C. Ferkinhoff et al., in preparation), we expected to find evidence of an AGN toward the compact blue source, but found instead that it is a star-our optical spectroscopy (Section 2.8) reveals absorption due to $\mathrm{Mg}$ as well as the $\mathrm{Na} \mathrm{D}$ doublet.

There is no obvious trend with wavelength for the relative IR/submm flux density contributions from $\mathrm{W}$ and $\mathrm{T}$ (listed in Table 1), $S_{\mathrm{W}} / S_{\mathrm{T}}=1.22 \pm 0.33,2.22 \pm 0.42,1.27 \pm 0.19,1.32 \pm$ $0.17,1.13 \pm 0.10$, and $1.23 \pm 0.25$ at $100 \mu \mathrm{m}, 160 \mu \mathrm{m}, 350 \mu \mathrm{m}$, $870 \mu \mathrm{m}, 1.36 \mathrm{~mm}$, and $2 \mathrm{~mm}$, respectively. The error-weighted mean for the IR/submm wavelength regime is $1.22 \pm 0.07$. Moving longward to rest frame $1.7 \mathrm{~cm}$, where we expect the emission to be dominated by synchrotron radiation related to supernova (SN) remnants, HATLAS J084933 is also dominated by component W (Figure 1), with $S_{\mathrm{W}} / S_{\mathrm{T}}=2.7 \pm 0.7$.

Continuum emission from dust is also seen east of $\mathrm{W}$, from a galaxy we label $\mathrm{M}$ in Figure 1. M is detected securely in $870 \mu \mathrm{m}$, $1.36 \mathrm{~mm}, 2.21 \mathrm{~mm}$, and $5.9 \mathrm{~cm}$ continuum (Table 1).

Still further to the east, emission can be seen from a very red, morphologically distorted galaxy-labeled $\mathrm{C}$ in Figure 1 -which bears a strong resemblance to the Antennae (e.g., Whitmore \& Schweizer 1995; Klaas et al. 2010). C is detected weakly in $870 \mu \mathrm{m}, 1.36 \mathrm{~mm}, 2.21 \mathrm{~mm}$, and $5.9 \mathrm{~cm}$ continuum (Table 1).

\footnotetext{
46 Data presented herein were obtained at the W. M. Keck Observatory, which is operated as a scientific partnership among the California Institute of Technology, the University of California, and NASA. The Observatory was made possible by the generous financial support of the W. M. Keck Foundation.
}

Table 1

Continuum Flux Densities

\begin{tabular}{|c|c|c|c|}
\hline \multirow[t]{2}{*}{ Wavelength } & \multicolumn{2}{|c|}{$S_{v}$} & \multirow[t]{2}{*}{ Facility } \\
\hline & $\mathrm{W}$ & $\mathrm{T}$ & \\
\hline $0.88 \mu \mathrm{m}$ & $8.16 \pm 1.37$ & $5.80 \pm 0.98$ & $\mu \mathrm{Jy} ;$ VISTA $Z$ \\
\hline $1.02 \mu \mathrm{m}$ & $10.5 \pm 1.8$ & $8.65 \pm 1.45$ & $\mu \mathrm{Jy} ;$ VISTA $Y$ \\
\hline $1.1 \mu \mathrm{m}$ & $13.6 \pm 2.3$ & $11.2 \pm 1.9$ & $\mu \mathrm{Jy} ; \mathrm{F} 110 \mathrm{~W}$ \\
\hline $1.25 \mu \mathrm{m}$ & $16.8 \pm 2.8$ & $13.0 \pm 2.2$ & $\mu \mathrm{Jy} ;$ VISTA $J$ \\
\hline $1.65 \mu \mathrm{m}$ & $23.5 \pm 4.0$ & $19.2 \pm 3.2$ & $\mu \mathrm{Jy} ;$ VISTA $H$ \\
\hline $2.15 \mu \mathrm{m}$ & $38.1 \pm 6.4$ & $26.1 \pm 4.4$ & $\mu \mathrm{Jy} ;$ VISTA $K_{\mathrm{s}}$ \\
\hline $3.6 \mu \mathrm{m}$ & $67.1 \pm 18.6$ & $47.7 \pm 17.4$ & $\mu \mathrm{Jy} ; \mathrm{IRAC}$ \\
\hline $4.5 \mu \mathrm{m}$ & $76.6 \pm 20.1$ & $45.8 \pm 15.2$ & $\mu \mathrm{Jy} ; \mathrm{IRAC}$ \\
\hline $100 \mu \mathrm{m}$ & $23 \pm 7^{\mathrm{a}}$ & $19 \pm 7^{\mathrm{a}}$ & mJy; PACS \\
\hline $160 \mu \mathrm{m}$ & $91 \pm 12^{\mathrm{a}}$ & $40 \pm 12^{\mathrm{a}}$ & mJy; PACS \\
\hline $250 \mu \mathrm{m}$ & \multicolumn{2}{|c|}{$242 \pm 18^{\mathrm{b}}$} & mJy; SPIRE \\
\hline $350 \mu \mathrm{m}$ & \multicolumn{2}{|c|}{$293 \pm 22^{b}$} & mJy; SPIRE \\
\hline $500 \mu \mathrm{m}$ & \multicolumn{2}{|c|}{$231 \pm 19^{b}$} & mJy; SPIRE \\
\hline $870 \mu \mathrm{m}$ & $25 \pm 2$ & $19 \pm 2$ & mJy; SMA \\
\hline $1.36 \mathrm{~mm}$ & $8.3 \pm 0.5$ & $7.5 \pm 0.5$ & mJy; PdBI \\
\hline $2.21 \mathrm{~mm}$ & $1.1 \pm 0.1$ & $0.8 \pm 0.1$ & mJy; PdBI \\
\hline $3.0 \mathrm{~mm}$ & $0.33 \pm 0.14$ & $3 \sigma<0.42$ & mJy; CARMA \\
\hline $8.8 \mathrm{~mm}$ & $0.043 \pm 0.010$ & $3 \sigma<0.030$ & mJy; JVLA \\
\hline \multirow[t]{2}{*}{$5.9 \mathrm{~cm}$} & $0.177 \pm 0.015$ & $0.065 \pm 0.015$ & mJy; JVLA \\
\hline & $\mathrm{C}$ & M & \\
\hline $0.88 \mu \mathrm{m}$ & $5.96 \pm 1.00$ & $\cdots$ & $\mu \mathrm{Jy} ;$ VISTA $Z$ \\
\hline $1.02 \mu \mathrm{m}$ & $5.46 \pm 0.92$ & $\cdots$ & $\mu \mathrm{Jy} ;$ VISTA $Y$ \\
\hline $1.1 \mu \mathrm{m}$ & $7.66 \pm 1.29$ & $\cdots$ & $\mu \mathrm{Jy} ; \mathrm{F} 110 \mathrm{~W}$ \\
\hline $1.25 \mu \mathrm{m}$ & $5.72 \pm 0.96$ & $\cdots$ & $\mu \mathrm{Jy} ;$ VISTA $J$ \\
\hline $1.65 \mu \mathrm{m}$ & $5.09 \pm 0.86$ & $\cdots$ & $\mu \mathrm{Jy} ;$ VISTA $H$ \\
\hline $2.15 \mu \mathrm{m}$ & $11.1 \pm 1.9$ & $\cdots$ & $\mu \mathrm{Jy} ;$ VISTA $K_{\mathrm{s}}$ \\
\hline $3.6 \mu \mathrm{m}$ & $19.5 \pm 5.9$ & $\cdots$ & $\mu \mathrm{Jy} ; \mathrm{IRAC}$ \\
\hline $4.5 \mu \mathrm{m}$ & $17.7 \pm 5.3$ & $\cdots$ & $\mu \mathrm{Jy} ; \mathrm{IRAC}$ \\
\hline $870 \mu \mathrm{m}$ & $4.6 \pm 1.9$ & $6.9 \pm 1.6$ & mJy; SMA \\
\hline $1.36 \mathrm{~mm}$ & $1.8 \pm 0.2$ & $1.5 \pm 0.2$ & mJy; PdBI \\
\hline $2.21 \mathrm{~mm}$ & $0.25 \pm 0.06$ & $0.31 \pm 0.08$ & mJy; PdBI \\
\hline $5.9 \mathrm{~cm}$ & $0.022 \pm 0.006$ & $0.020 \pm 0.005$ & mJy; JVLA \\
\hline
\end{tabular}

Notes.

${ }^{a}$ Errors determined by placing many apertures across the map, with $3 \%$ and $5 \%$ calibration uncertainties added in quadrature at 100 and $160 \mu \mathrm{m}$, respectively.

b Errors include the contribution due to confusion and a $7 \%$ calibration uncertainty has been added in quadrature (E. Valiante et al., in preparation).

As we shall see in more detail later (Section 3.5), $\mathrm{C}$ and $\mathrm{M}$ are also detected in our CO $J=1-0$ and $J=4-3$ spectral-line imaging, i.e., they also lie at $z=2.41$, alongside $\mathrm{W}$ and $\mathrm{T}$.

Both $\mathrm{M}$ and $\mathrm{C}$ are coincident with IRAC 3.6-4.5 $\mu \mathrm{m}$ emission, suggesting that these are not tidal tails or gaseous streams, but rather significant concentrations of stars, gas, and dust-luminous SMGs in their own right.

Our deep rest-frame 133-289 nm Keck spectroscopy (Section 2.8), with slits covering W, T, and C, reveals no significant line emission from $\mathrm{T}$ and $\mathrm{C}$; the only line visible from $\mathrm{W}$, albeit very faint, is $\mathrm{C}_{\mathrm{II}}$ at $232.6 \mathrm{~nm}$.

Our CO $J=3-2$ image from CARMA is shown in Figure 1. It is interesting to note that the low-level southern extension to the $J=3-2$ emission from $\mathrm{T}$ is mirrored at 3.6-4.5 $\mu \mathrm{m}$, which suggests that both are real.

\subsection{Lensing Model}

The lenticular galaxy that lies along the line of sight to component $\mathrm{T}$ lies at $z=0.3478$, as revealed by our optical spectroscopic observations (Section 2.8), where the slit was placed along the major axis of the lenticular. Correcting for 


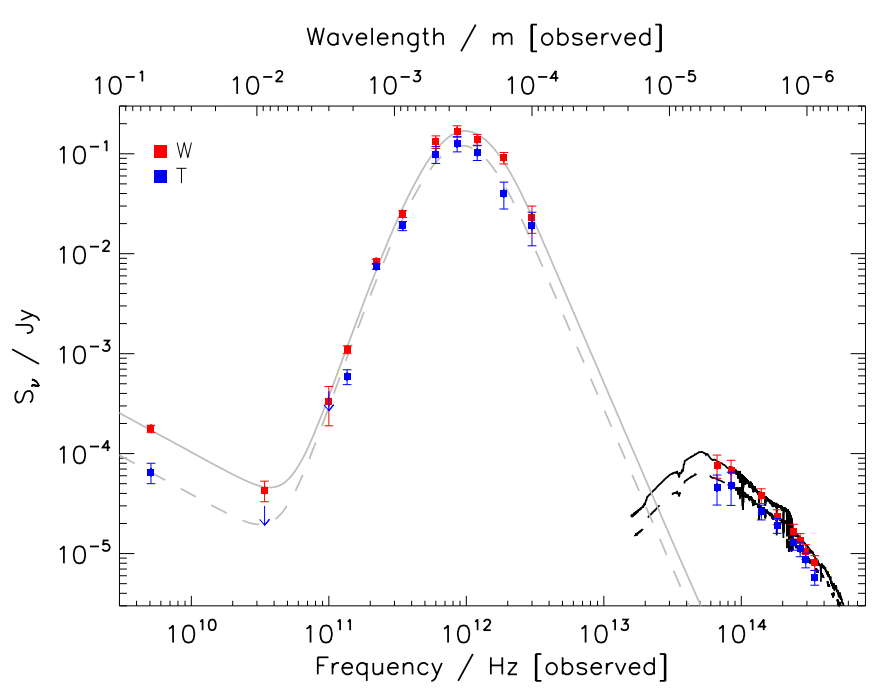

Figure 2. Radio-through-optical SEDs of the dominant components of HATLAS J084933, W (red points, solid line) and T (blue points, dotted line). Our measurements cover the SED peak, constraining $L_{\mathrm{IR}}$ and $T_{\text {dust }}$ well. The SEDs can be fitted adequately using a combination of thermal dust and synchrotron emission components (see Section 3.3) plus the stellar population modeling described in Section 3.4. The key resulting characteristics, $L_{\mathrm{IR}}, T_{\mathrm{dust}}, M_{\mathrm{dust}}, M_{\mathrm{stars}}$, and $q_{\mathrm{IR}}$ are listed in Table 2 .

(A color version of this figure is available in the online journal.)

the instrumental resolution, the lines due to $\mathrm{Mg}$ and $\mathrm{Ca} \mathrm{H}$ and $\mathrm{K}$ suggest the foreground galaxy has a velocity dispersion of $190 \pm 80 \mathrm{~km} \mathrm{~s}^{-1}$. Its stellar mass is $(1.1 \pm 0.4) \times 10^{11} M_{\odot}$, adopting the approach outlined later in Section 3.4. We have modeled this galaxy with an elliptical $(e=0.3)$ mass profile, truncated smoothly at a radius of $25 \mathrm{kpc}$ (the exact choice has little effect since the lensed image is well within this radius). The line of sight to the $z=2.41$ starburst lies close to the semimajor axis of this foreground structure and suffers a magnification of $\mu=1.5 \pm 0.2$, where the uncertainty was calculated by varying the model parameters-orientation, ellipticity, and velocity dispersion-by $10 \%$.

Galaxy W suffers no significant lensing magnification, as far as it is possible to discern from our extensive, wide-field, panchromatic imaging data (Section 2.7), which are not easily reconciled with a massive foreground galaxy group or cluster.

\subsection{Spectral Energy Distributions of $W$ and $T$}

Figure 2 shows the spectral energy distribution (SED) of HATLAS J084933, concentrating on the brightest components, $\mathrm{W}$ and $\mathrm{T}$. Our CSO/SHARC-2 $350 \mu \mathrm{m}$ imaging provides evidence that - at the peak of their SEDs - the relative SPIRE contributions of components $\mathrm{W}$ and $\mathrm{T}$ follow the average ratio seen in the resolved imaging, $1.22 \pm 0.07$, as described in Section 3.1. Perhaps unsurprisingly, then, we then find they contribute in roughly the same ratio, $1.55 \pm 0.44$, to an immense overall bolometric luminosity of HATLAS J084933, $L_{\mathrm{IR}}=(4.8 \pm 1.0) \times 10^{13} L_{\odot}($ Table 2$)$, having corrected the contribution from $T$ for gravitational amplification (Section 3.2).

The IR-through-radio photometry can be described adequately with a model comprising synchrotron and thermal dust emission, following Kovács et al. (2010). Table 2 summarizes a simultaneous fit to the dominant cold dust temperature, $T_{\text {dust }}$, to $L_{\mathrm{IR}}$ and $q_{\mathrm{IR}}$ (as defined by Helou et al. 1985, but where $S_{\mathrm{IR}}$ is measured across $\lambda_{\text {rest }}=8-1000 \mu \mathrm{m}$, as is $L_{\mathrm{IR}}$ ), and to $M_{\text {dust }}$ (for a characteristic photon cross-section to mass ratio, $\kappa_{850}=0.077 \mathrm{~m}^{2} \mathrm{~kg}^{-1}$-Dunne et al. 2000; Dunne
$\&$ Eales 2001-where we fixed the frequency dependence of the dust emissivity, $\beta$, to be 2.0 , which is physically plausible and consistent with the best-fit value, $2.08 \pm 0.15)$. We fixed the synchrotron power-law index, $\alpha=-0.75$, where $S_{v} \propto v^{\alpha}$, and we also fixed $\gamma=7.2$ - the power-law index of a dust temperature distribution appropriate for local starbursts, $d M_{\text {dust }} / d T_{\text {dust }} \propto T_{\text {dust }}^{-\gamma}$, which is designed to offer a physically motivated treatment of the Wien side of the thermal emission spectrum. Both $\mathrm{W}$ and $\mathrm{T}$ have $T_{\text {dust }} \sim 36-40 \mathrm{~K}$, commensurate with similarly luminous, dusty starbursts at $z \sim 2-3$, when calculated using a power-law temperature distribution (Magnelli et al. 2012).

We are sensitive to AGN activity via our optical imaging and spectroscopy (Section 2.8) and our radio/mid-IR imaging (Sections 2.1 and 2.7). In relation to the far-IR/radio flux ratio, $\mathrm{W}$ and $\mathrm{T}$ have $q_{\mathrm{IR}}=2.30 \pm 0.08$ and $2.53 \pm 0.13$, respectively, spanning (and consistent with) the tight correlation for starforming galaxies (SFGs; $q_{\mathrm{IR}} \approx 2.4$, e.g., Yun et al. 2001). Summarizing, we have no evidence ${ }^{47}$ that an AGN contributes significantly to the $L_{\mathrm{IR}}$ of HATLAS J084933, though of course we have no evidence of absence since even a powerful AGN can be hidden very effectively by the quantities of dust-rich gas in this system.

If contributions to $L_{\mathrm{IR}}$ from $\mathrm{AGNs}$ are small, then $\mathrm{W}$ and $\mathrm{T}$ are generating stars at a rate of $\approx 3400$ and $\approx 1500 M_{\odot} \mathrm{yr}^{-1}$, respectively, for a Chabrier (2003) IMF (Kennicutt 1998, though see Dwek et al. 2011). The estimates of $L_{\mathrm{IR}}$ for $\mathrm{C}$ and $\mathrm{M}$ given in Table 2 are scaled from their submm photometry relative to $\mathrm{W}$ and $\mathrm{T}$, assuming the same SED shape. This suggests that both are close to being HyLIRGs in their own right, $\approx 7 \times 10^{12} L_{\odot}$.

Before closing our SED discussion, we add a word of caution regarding our estimated IR luminosities. If $\mathrm{W}$ and $\mathrm{T}$ lie in the core of a protocluster, as we shall see in what follows, then lowerluminosity star-forming cluster galaxies may contribute to the flux densities measured by the relatively large SPIRE beam at 250-500 $\mu \mathrm{m}$ (see Negrello et al. 2005). Although the calibration cannot compare with that of Herschel, SHARC-2 recovers less flux than SPIRE at $350 \mu \mathrm{m}$, a possible manifestation of the protocluster.

\subsection{Stellar Masses}

The rest-frame ultraviolet-through-near-IR SEDs of $\mathrm{W}$ and $\mathrm{T}$ are illustrated in Figure 2, alongside the far-IR-submm-radio data. Flux densities are listed in Table 1. Our high-quality HST, VIKING, and IRAC images at $0.88-4.5 \mu \mathrm{m}$ are compromised to a certain extent by the unfortunate super-position of a star and a lenticular galaxy near W and T, respectively, but the considerable uncertainties associated with estimating stellar mass dominate over the photometric uncertainties (see, e.g., Hainline et al. 2011; Michalowski et al. 2012).

For components $\mathrm{W}, \mathrm{T}$, and $\mathrm{C}$, we find best-fitting monochromatic rest-frame $H$-band luminosities, $\log L_{\mathrm{H}}$, of $11.82,11.46$, and $11.12 L_{\odot}$, respectively. Stellar masses were determined using MAGPHYS with the most recent version of the stellar population models of Bruzual \& Charlot (2003), a Chabrier (2003) IMF, and the SED prior distributions described by da Cunha et al. (2008), following Rowlands et al. (2012). The models encompass a wide range of exponentially declining star formation histories (SFHs), with bursts superimposed (see da Cunha et al. 2008 for details). The implied stellar masses are

\footnotetext{
47 Observations of higher- $J$ CO lines would be useful in terms of AGN
} diagnostics. 
Table 2

Properties of HATLAS J084933.4+021443

\begin{tabular}{|c|c|c|c|c|}
\hline Property & $\mathrm{W}$ & $\mathrm{T}$ & M & $\mathrm{C}$ \\
\hline R.A. (J2000) & $08: 49: 33.59$ & $08: 49: 32.96$ & $08: 49: 33.80$ & $08: 49: 33.91$ \\
\hline Decl. (J2000) & $+02: 14: 44.6$ & $+02: 14: 39.7$ & $+02: 14: 45.6$ & $+02: 14: 45.0$ \\
\hline Mean FWHM at $5.9 \mathrm{~cm}, 870 \mu \mathrm{m}$, and in CO $J=4-3$ & $2.9 \pm 0.4 \mathrm{kpc}$ & $3.8 \pm 0.6 \mathrm{kpc}^{\mathrm{a}}$ & $\mathrm{b}$ & $\mathrm{b}$ \\
\hline FWHM in CO $J=1-0(\mathrm{kpc})$ & $7.0 \pm 2.1 \mathrm{kpc}$ & $6.2 \pm 1.4 \mathrm{kpc}^{\mathrm{a}}$ & b & b \\
\hline $\log L_{\mathrm{IR}}\left(L_{\odot}\right)$ & $13.52 \pm 0.04$ & $13.16 \pm 0.05^{\mathrm{a}}$ & $12.9 \pm 0.2$ & $12.8 \pm 0.2$ \\
\hline SFR $\left(M_{\odot} \mathrm{yr}^{-1}\right)($ Kennicutt 1998; Chabrier 2003 IMF) & 3400 & $1500^{\mathrm{a}}$ & 800 & 640 \\
\hline$q_{\mathrm{IR}}$ & $2.30 \pm 0.08$ & $2.53 \pm 0.13$ & b & b \\
\hline $\log M_{\text {dust }}\left(M_{\odot}\right)$ & $9.32 \pm 0.05$ & $9.10 \pm 0.05^{\mathrm{a}}$ & $\mathrm{b}$ & $\mathrm{b}$ \\
\hline $\mathrm{CO} J=1-0 I_{\mathrm{CO}}\left(\mathrm{Jy} \mathrm{km} \mathrm{s}^{-1}\right)$ & $0.49 \pm 0.06$ & $0.56 \pm 0.07$ & $0.057 \pm 0.013$ & $0.079 \pm 0.014$ \\
\hline $\mathrm{CO} J=1-0 L_{\mathrm{CO}}^{\prime}\left(10^{9} \mathrm{~K} \mathrm{~km} \mathrm{~s}^{-1} \mathrm{pc}^{2}\right)$ & $138 \pm 17$ & $157 \pm 20$ & $16.0 \pm 3.6$ & $22.2 \pm 3.9$ \\
\hline $\mathrm{CO} J=1-0 L_{\mathrm{CO}}\left(10^{6} L_{\odot}\right)$ & $6.72 \pm 0.82$ & $7.65 \pm 0.96$ & $0.779 \pm 0.178$ & $0.108 \pm 0.019$ \\
\hline $\mathrm{CO} J=1-0 \mathrm{FWHM}\left(\mathrm{km} \mathrm{s}^{-1}\right)$ & $825 \pm 115$ & $610 \pm 55$ & $320 \pm 70$ & $250 \pm 100$ \\
\hline $\mathrm{CO} J=1-0 z_{\mathrm{LSR}}$ & $2.4066 \pm 0.0006$ & $2.4090 \pm 0.0003$ & $2.4176 \pm 0.0004$ & $2.4138 \pm 0.0003$ \\
\hline $\mathrm{CO} J=3-2 I_{\mathrm{CO}}\left(\mathrm{Jy} \mathrm{km} \mathrm{s}^{-1}\right)$ & $4.08 \pm 0.92$ & $5.73 \pm 1.13$ & $0.66 \pm 0.27$ & $1.16 \pm 0.37$ \\
\hline $\mathrm{CO} J=3-2 \mathrm{FWHM}\left(\mathrm{km} \mathrm{s}^{-1}\right)$ & $830 \pm 155$ & $555 \pm 75$ & $260 \pm 95$ & $620 \pm 160$ \\
\hline $\mathrm{CO} J=3-2 z_{\mathrm{LSR}}$ & $2.4077 \pm 0.0009$ & $2.4096 \pm 0.0004$ & $2.4173 \pm 0.0006$ & $2.414^{\mathrm{d}}$ \\
\hline $\mathrm{CO} J=4-3 I_{\mathrm{CO}}\left(\mathrm{Jy} \mathrm{km} \mathrm{s}^{-1}\right)$ & $3.76 \pm 0.27$ & $5.10 \pm 0.37$ & $0.66 \pm 0.10$ & $0.95 \pm 0.15$ \\
\hline $\mathrm{CO} J=4-3 \mathrm{FWHM}\left(\mathrm{km} \mathrm{s}^{-1}\right)$ & $985 \pm 115$ & $545 \pm 30$ & $320 \pm 50$ & $450 \pm 90$ \\
\hline $\mathrm{CO} J=4-3 z_{\mathrm{LSR}}$ & $2.4068 \pm 0.0002$ & $2.4090 \pm 0.0002$ & $2.4178 \pm 0.0003$ & $2.4149 \pm 0.0004$ \\
\hline$T_{\mathrm{b}} r_{3-2 / 1-0}$ & $0.93 \pm 0.24$ & $1.14 \pm 0.27$ & $1.29 \pm 0.60$ & $1.63 \pm 0.59$ \\
\hline$T_{\mathrm{b}} r_{4-3 / 1-0}$ & $0.48 \pm 0.07$ & $0.57 \pm 0.08$ & $0.72 \pm 0.20$ & $0.75 \pm 0.18$ \\
\hline$v_{\max }\left(\mathrm{km} \mathrm{s}^{-1}\right)$ & $350 \pm 10$ & $220 \pm 15$ & $20 \pm 10$ & $80 \pm 20$ \\
\hline$R(\mathrm{kpc})$ & $7.8 \pm 1.0$ & $6.8 \pm 0.8^{\mathrm{a}}$ & b & b \\
\hline $\log M_{\text {dyn }}\left(M_{\odot}\right)$ & $11.51 \pm 0.05$ & $11.13 \pm 0.05^{\mathrm{a}}$ & $11.11 \pm 0.15$ & $11.11 \pm 0.20$ \\
\hline $\log M_{\mathrm{H}_{2}+\mathrm{He}}\left(M_{\odot}\right)$, for $\alpha_{\mathrm{CO}}=0.8 M_{\odot}\left(\mathrm{K} \mathrm{km} \mathrm{s}^{-1} \mathrm{pc}^{2}\right)^{-1}$ & $11.04 \pm 0.05$ & $10.92 \pm 0.06^{\mathrm{a}}$ & $10.11 \pm 0.09$ & $10.25 \pm 0.07$ \\
\hline $\operatorname{SFE}\left(L_{\odot} \mathbf{M}_{\odot}^{-1}\right)$ & 370 & $210^{\mathrm{a}}$ & 760 & 440 \\
\hline $\log M_{\text {stars }}\left(M_{\odot}\right)$ & $11.38 \pm 0.12$ & $11.01 \pm 0.12^{\mathrm{a}}$ & $\approx 10$ & $10.36 \pm 0.18$ \\
\hline
\end{tabular}

Notes.

${ }^{\text {a }}$ Corrected for $\mu=1.5 \pm 0.2$.

${ }^{\mathrm{b}}$ Insufficient $\mathrm{S} / \mathrm{N}$ to make a useful measurement.

c Adopting $S_{\text {total }} / S_{\text {peak }}$ as seen for ${ }^{12} \mathrm{C}^{16} \mathrm{O}$.

${ }^{\mathrm{d}}$ Fixed.

$\log M_{\text {stars }}=11.38_{-0.13}^{+0.11}, 11.01_{-0.11}^{+0.12}$ and $10.36_{-0.17}^{+0.18} M_{\odot}$ for W, T, and $\mathrm{C}$, where the errors are derived from the marginalized probability density function, which incorporates the uncertainties in the $\mathrm{SFH}^{48}$ and photometry. Systematic shifts in stellar mass of $\approx 0.2$ dex result from adopting a Salpeter IMF or ignoring thermally pulsating stars on the asymptotic giant branch, and the magnitude of photometric contamination by buried AGNs could well be similar, so we estimate that our stellar mass estimates are accurate to $\approx 0.3$ dex.

Emission from $\mathrm{M}$ can be seen at 3.6-4.5 $\mu \mathrm{m}$ but the photometric uncertainties due to blending with $\mathrm{C}$ and $\mathrm{W}$ are large so we state only that its stellar mass must be considerable, $\approx 10^{10} M_{\odot}$.

There is little sign of the power-law SEDs that can betray the presence of AGNs, supporting the lack of AGN spectral features reported in Section 3.1, though of course this does not rule out the presence of a deeply buried, highly obscured AGN.

\subsection{Spectral-line Characteristics}

Figure 3 shows the JVLA CO $J=1-0$ spectra of $\mathrm{W}$ and T alongside our IRAM CO $J=4-3$ spectra. These spectral-line data bring us velocity information for the first time, enabling us to see that although $\mathrm{W}$ and $\mathrm{T}$ do lie at approximately the same

\footnotetext{
48 Stellar mass estimates are generally robust to changes in SFH (e.g., Ilbert
} et al. 2010, 2013; Pforr et al. 2012). redshift and share similar line profiles and $T_{\mathrm{b}}$ ratios, they have significantly different line widths (Table 2). Each one is thus a distinct, extraordinarily luminous starburst. While the $\mathrm{CO}$ line profile of T is typical of SMGs (e.g., Bothwell et al. 2013), that of $\mathrm{W}$ is broader than most, with $\approx 1000 \mathrm{~km} \mathrm{~s}^{-1}$ FWZI. Both profiles are flat-topped or perhaps double-peaked; they cannot be described well by a single Gaussian.

The CO $J=1-0$ fluxes of $\mathrm{W}$ and T measured in our JVLA data sum to $I_{\mathrm{CO} 1-0}=1.05 \pm 0.09 \mathrm{Jy} \mathrm{km} \mathrm{s}{ }^{-1}$. At first sight this is consistent with the Harris et al. GBT measurement, $1.04 \pm 0.37 \mathrm{Jy} \mathrm{km} \mathrm{s}^{-1}$. However, the CO $J=1-0$ line profiles observed by JVLA and GBT appear noticeably different in Figure 3: emission in the GBT spectrum continues significantly redward (by $\approx 500 \mathrm{~km} \mathrm{~s}^{-1}$ ) of the emission that can attributed to $\mathrm{W}$ and $\mathrm{T}$.

Looking at our IRAM PdBI imaging-see Figure 4-after subtracting the significant continuum emission (several submm continuum flux densities are listed in Table 1), we see that both $\mathrm{C}$ and $\mathrm{M}$ are detected securely in CO $J=4-3$ (at $9.0 \sigma$ and $7.2 \sigma$, respectively; we also see $3.6-4.5 \mu \mathrm{m}$, submm, and radio continuum emission-recall Figure 1). These galaxies are therefore unquestionably real and they share approximately the same redshift as components $\mathrm{W}$ and $\mathrm{T}$. Their positions are listed in Table 2, along with those of the two brighter components. Their $\mathrm{CO}$ emission is centered $\approx 500 \mathrm{~km} \mathrm{~s}^{-1}$ redward of $\mathrm{W}$ and $\mathrm{T}$. 

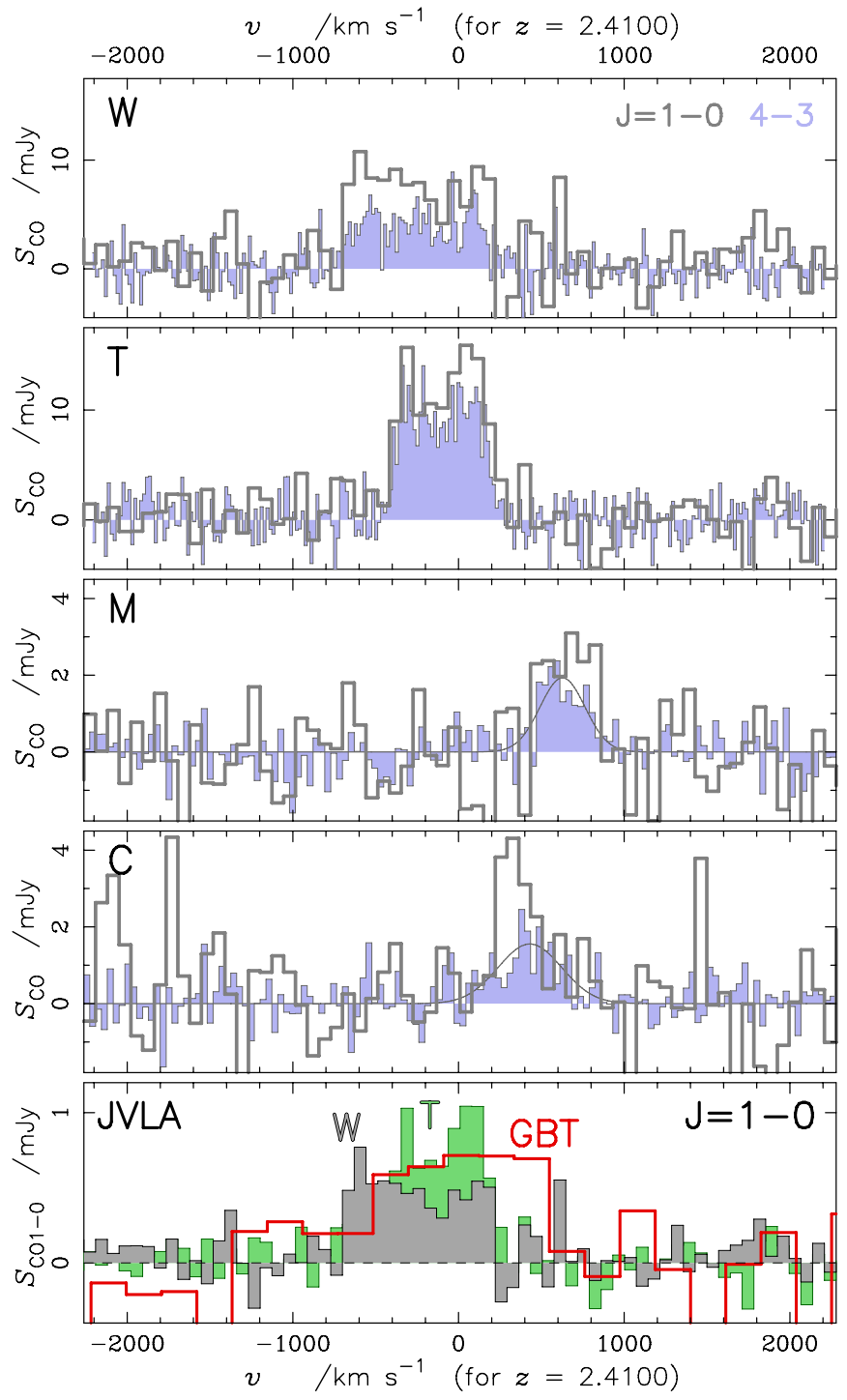

Figure 3. CO spectra of HATLAS J084933. Upper two panels: CO $J=1-0$ spectra of galaxies $\mathrm{W}$ and $\mathrm{T}$ superimposed on solid representations of the CO $J=4-3$ spectra (the former scaled by $16 \times$ to put them on the same $T_{\mathrm{b}}$ scale). The two galaxies both have extraordinarily broad $\mathrm{CO}$ lines but the profiles are noticeably different, so they are not lensed images of the same galaxy. The profiles of the $\mathrm{CO}$ transitions are indistinguishable for each individual component; their $T_{\mathrm{b}}$ ratios are also similar (Table 2). Middle two panels: CO $J=4-3$ spectra of galaxies $\mathrm{M}$ and $\mathrm{C}$, with line centers significantly redward of $\mathrm{W}$ and T. Lower panel: JVLA CO $J=1-0$ spectra of $\mathrm{W}$ and $\mathrm{T}$ alongside the significantly different profile from GBT/Zpectrometer (Harris et al. 2012). Emission at velocities redward of $\mathrm{W}$ and $\mathrm{T}-$ from $\mathrm{M}$ and $\mathrm{C}$, and several other faint clumps seen in the JVLA data, spanning a $\sim 100 \mathrm{kpc}$ region-can account for the differences between the GBT and JVLA line profiles.

(A color version of this figure is available in the online journal.)

As well as $\mathrm{C}$ and $\mathrm{M}$, a number of other faint emission features can seen in the integrated $\mathrm{CO} J=1-0$ image (Figure 1). Summing $\mathrm{C}, \mathrm{M}$, and these faint clumps leads to tolerable agreement between the GBT and JVLA line profiles. Although we must add $0.87 \pm 0.19 \mathrm{Jy} \mathrm{km} \mathrm{s}^{-1}$ to $I_{\mathrm{CO}}^{\mathrm{JVLA}}$, we must also increase $I_{\mathrm{CO}}^{\mathrm{GBT}}$ by $\approx 15 \%$ to account for the $\sim 8 \%$ lower flux density assumed for 3C 286 by Harris et al. (2012), and for losses due to attenuation of the faint clumps by the GBT primary beam. The agreement ${ }^{49}$ between the total GBT and JVLA line

\footnotetext{
49 Note that the low-level GBT spectrometer baseline (at negative velocities relative to HATLAS J084933) may have caused an underestimate of $I_{\mathrm{CO}}^{\mathrm{GBT}}$.
}

intensities remains consistent to within $\approx 1 \sigma$ and the profile measured by the FWHM $\approx 22^{\prime \prime}$ GBT primary beam is thereby reconciled with the JVLA data.

\subsection{Resolved Imaging of the Gas and Dust}

Our interferometric images from JVLA, IRAM PdBI, and the SMA have a spatial resolution of $\sim 0.5 \mathrm{FWHM}$, or $\sim 4 \mathrm{kpc}$. They allow us to compare the sizes of the regions responsible for emission from the total molecular gas reservoir (via CO $J=1-0$ emission), from the star-forming gas (via $\mathrm{CO} J=4-3$ ), from cool dust produced by $\mathrm{SNe}$ in regions of recent star formation (via submm continuum emission) and from relativistic electrons spiraling in or near recent $\mathrm{SN}$ remnants (via radio continuum emission).

We find that emission from $\mathrm{W}$ and $\mathrm{T}$ is resolved in all these wavebands, with total flux densities significantly higher than the peak flux densities. In Table 2 we quote their deconvolved FWHM $^{50}$ sizes at $5.9 \mathrm{~cm}, 870 \mu \mathrm{m}$ and in CO $J=4-3$ and $J=1-0$, as determined with two-dimensional (2D) Gaussian fits.

In the absence of significant AGN-powered activity, radio continuum observations trace regions where massive stars have recently been formed, with no obscuration. Of the tracers available to us, we might anticipate the radio emission to be the most compact, as indeed is measured. At $870 \mu \mathrm{m}$ we see no evidence of a more extended emission component, heated by older stars - the deconvolved sizes of $\mathrm{W}$ and $\mathrm{T}$ are consistent, as measured in $5.9 \mathrm{~cm}$ and $870 \mu \mathrm{m}$ continuum, as one might expect given the far-IR/radio correlation (Table 2). The CO $J=4-3$ FWHM are slightly larger, as seen for local ultraluminous infrared galaxies (ULIRGs; e.g., Wilson et al. 2008), but are consistent with the radio and submm values to within the uncertainties.

The error-weighted average of the $5.9 \mathrm{~cm}$ and $870 \mu \mathrm{m}$ continuum and CO $J=4-3$ FWHM measurements is $(3.4 \pm$ $0.4) \mathrm{kpc} \times(2.4 \pm 0.4) \mathrm{kpc}\left(\right.$ P.A., $\left.9 \pm 15^{\circ}\right)$ for $\mathrm{W}$; for $\mathrm{T}$, before correcting for the amplification, we measure $(5.6 \pm 0.7) \mathrm{kpc} \times$ $(3.5 \pm 0.7) \mathrm{kpc}\left(\right.$ P.A., $\left.48 \pm 16^{\circ}\right)$. In CO $J=1-0$, however, W and $\mathrm{T}$ have considerably larger deconvolved sizes than those measured in the submm or radio continuum, as seen for SMGs generally (e.g., Ivison et al. 2011; Riechers et al. 2011).

Summarizing, we have powerful, direct evidence of starbursts covering $\approx 3-4 \mathrm{kpc}$ FWHM, with still larger reservoirs of gas available to fuel future star formation, on scales of $\approx 6-7 \mathrm{kpc}$ FWHM.

The large sizes measured in $\mathrm{CO}$ have been mentioned alongside the relatively high $L_{[\mathrm{C} \text { II] }} / L_{\mathrm{IR}}$ ratios measured toward a number of SMGs (e.g., Stacey et al. 2010). Both have been taken as evidence that SMGs form stars across larger spatial scales than the compact, nuclear events seen in local ULIRGs.

\subsection{Dynamical Characteristics}

The most remarkable characteristics of HATLAS J084933 are revealed by the superlative spatial resolution (Section 3.6), and velocity resolution and sensitivity of our JVLA CO $J=1-0$ and IRAM PdBI CO $J=4-3$ data, as shown in Figure 4.

To measure the $2 \mathrm{D}$ velocity structure, we fit the $\mathrm{CO}$ emission lines, pixel by pixel, in each of the cubes. Initially, we attempt

\footnotetext{
50 We use half-light radii to calculate the area appropriate for gas and star formation surface densities, alongside a $0.5 \times$ correction to the SFR or gas mass. We use FWHM simply as a convenient way to compare sizes in several wavebands.
} 


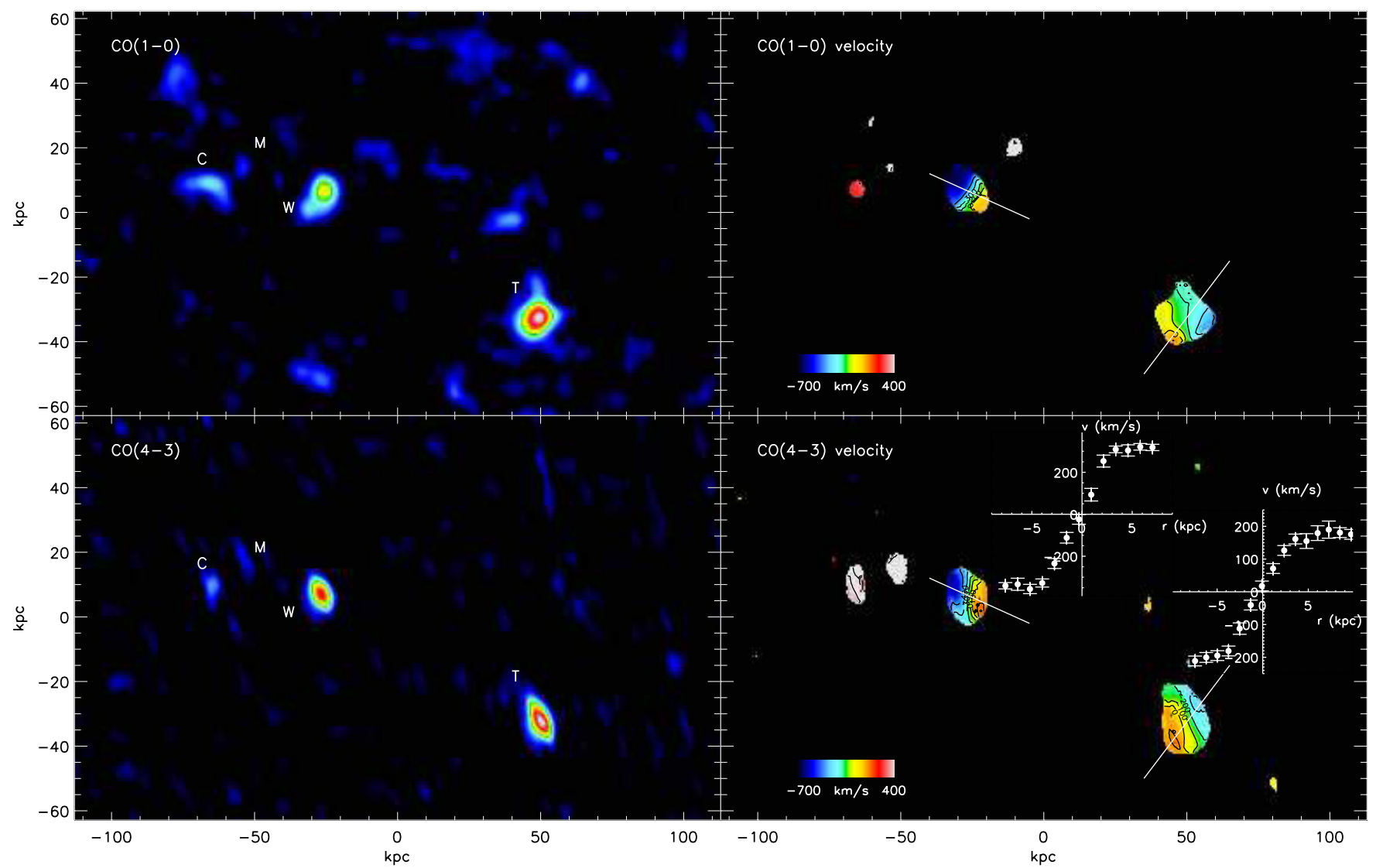

Figure 4. CO $J=1-0$ and $J=4-3$ imaging of HATLAS J084933. Left: velocity-integrated continuum-subtracted CO emission from our JVLA and PdBI observations. In both panels, we mark the positions of the brightest four galaxies, $\mathrm{C}, \mathrm{M}, \mathrm{W}$, and T. Right: dynamical maps of the galaxies in CO $J=1-0$ and $J=4-3$. To construct these velocity fields, we fit the $\mathrm{CO}$ emission in each cube at each pixel, recording the intensity, velocity, and line width where $\mathrm{S} / \mathrm{N}>5$. North is up and east is to the left. Inset: one-dimensional rotation curves for $\mathrm{W}$ and $\mathrm{T}$, extracted along the major kinematic axis identified in the modeling. Both $\mathrm{W}$ and $\mathrm{T}$ have rotation curves which resemble disks; indeed, W and T are best described as counter-rotating disks, which Mihos \& Hernquist (1996) predicted would lead to the most intense starbursts.

(A color version of this figure is available in the online journal.)

to identify an emission line in $0.2 \times 0.2$ regions, increasing this slowly until we reach $\mathrm{S} / \mathrm{N}>5$, at which point we fit the $\mathrm{CO}$ profile, allowing the intensity, centroid, and width to vary. Uncertainties are calculated by perturbing each parameter independently, allowing the remaining parameters to find their optimum values, until $\Delta \chi^{2}=1$ is reached.

We model the 2D velocity fields of $\mathrm{W}$ and $\mathrm{T}$ by constructing and fitting 2D disk models using an arctan function, $v(r)=$ $2 \pi^{-1} v_{\text {asym }} \arctan \left(r / r_{t}\right)$, to describe the kinematics (where $v_{\text {asym }}$ is the asymptotic inclination-corrected rotational velocity and $r_{\mathrm{t}}$ is the effective radius at which the rotation curve turns over-see Courteau 1997). We fit six free parameters to the disk velocity field: $v_{\text {asym }}, r_{\mathrm{t}}, x, y$ centroid, P.A., and inclination to the sky. The $x, y$ centroid, and $r_{\mathrm{t}}$ must be within the field of view and the maximum circular velocity must be $<2$ times the maximum velocity seen in the data. Quoted uncertainties reflect the range of acceptable models from all of the attempted model fits.

Our velocity cubes reveal that the wide $\mathrm{CO}$ line that led to this system being selected as a candidate HyLIRG is due partly to the multitude of constituent galaxies and partly to large regular, systematic rotational velocities in the two brightest constituent galaxies. Both $\mathrm{W}$ and $\mathrm{T}$ are resolved into counter-rotating gas disks - the scenario that many studies have predicted should lead to the most intense starbursts (e.g., Mihos \& Hernquist 1994, 1996; Taniguchi \& Shioya 1998; Borne et al. 2000; Bekki 2001; Di Matteo et al. 2007; Salomé et al. 2012).
As shown in Figure 5, we find acceptable fits for $\mathrm{W}$ and $\mathrm{T}$ with disk inclinations of $56^{\circ} \pm 10^{\circ}$ and $49^{\circ} \pm 10^{\circ}$, at P.A.s of $26^{\circ}$ and $145^{\circ}$, with $v_{\text {asym }}=415$ and $370 \mathrm{~km} \mathrm{~s}^{-1}$, respectively. Component $\mathrm{T}$ is sheared slightly by the foreground lens, as evident from the velocity and $\sigma$ maps. Both galaxies show small-scale (30-60 $\mathrm{km} \mathrm{s}^{-1}$, rms) deviations from the bestfit model. The dynamical masses ${ }^{51}$ of $\mathrm{W}$ and $\mathrm{T}$ are listed in Table 2: several $\times 10^{11} M_{\odot}$, where $M_{\mathrm{dyn}}=R v_{\max }^{2} / \sin ^{2}(i)$. Their velocity dispersions, $\sigma$, corrected for the instrumental velocity resolution, are $\approx 65 \mathrm{~km} \mathrm{~s}^{-1}$.

$\mathrm{M}$ has no strong velocity gradient. Its $v_{\max }$ is $20 \mathrm{~km} \mathrm{~s}^{-1}$ (40 $\mathrm{km} \mathrm{s}^{-1}$, peak to peak), $\sigma=140 \pm 15 \mathrm{~km} \mathrm{~s}^{-1}$ for both CO $J=1-0$ and $J=4-3$, and $M_{\text {dyn }} \approx 1.3 \times 10^{11} M_{\odot}$. For C, in CO $J=1-0$ we find $v_{\max }=80 \mathrm{~km} \mathrm{~s}^{-1}\left(160 \mathrm{~km} \mathrm{~s}^{-1}\right.$, peak to peak), $\sigma=130 \pm 20 \mathrm{~km} \mathrm{~s}^{-1}$, and $M_{\text {dyn }} \approx 1.3 \times 10^{11} M_{\odot}$ (in CO $J=4-3$ we find slightly higher values for $\sigma$ and $M_{\text {dyn }}$ ).

\subsection{Star Formation in Disks Off the Main Sequence}

For main-sequence galaxies, star formation is not confined to a compact starbursting nucleus (e.g., Elbaz et al. 2011); at $z \sim$ 2.4, the specific SFR (sSFR) is approximately $2.8 \mathrm{Gyr}^{-1}$. W and T have sSFRs of 14.2 and $14.7 \mathrm{Gyr}^{-1}$, so their starburstiness, ${ }^{52}$ $\mathrm{sSFR} / \mathrm{sSFR}_{\mathrm{MS}} \gtrsim 5$, placing them well above the main sequence.

\footnotetext{
$51 R$ was taken to be twice the deconvolved half-light radius in CO $J=1-0$. 52 We cannot determine the $I R 8$ starburst indicator (Elbaz et al. 2011) for HATLAS J084933 since we lack sufficiently deep rest-frame $8 \mu \mathrm{m}$ imaging.
} 

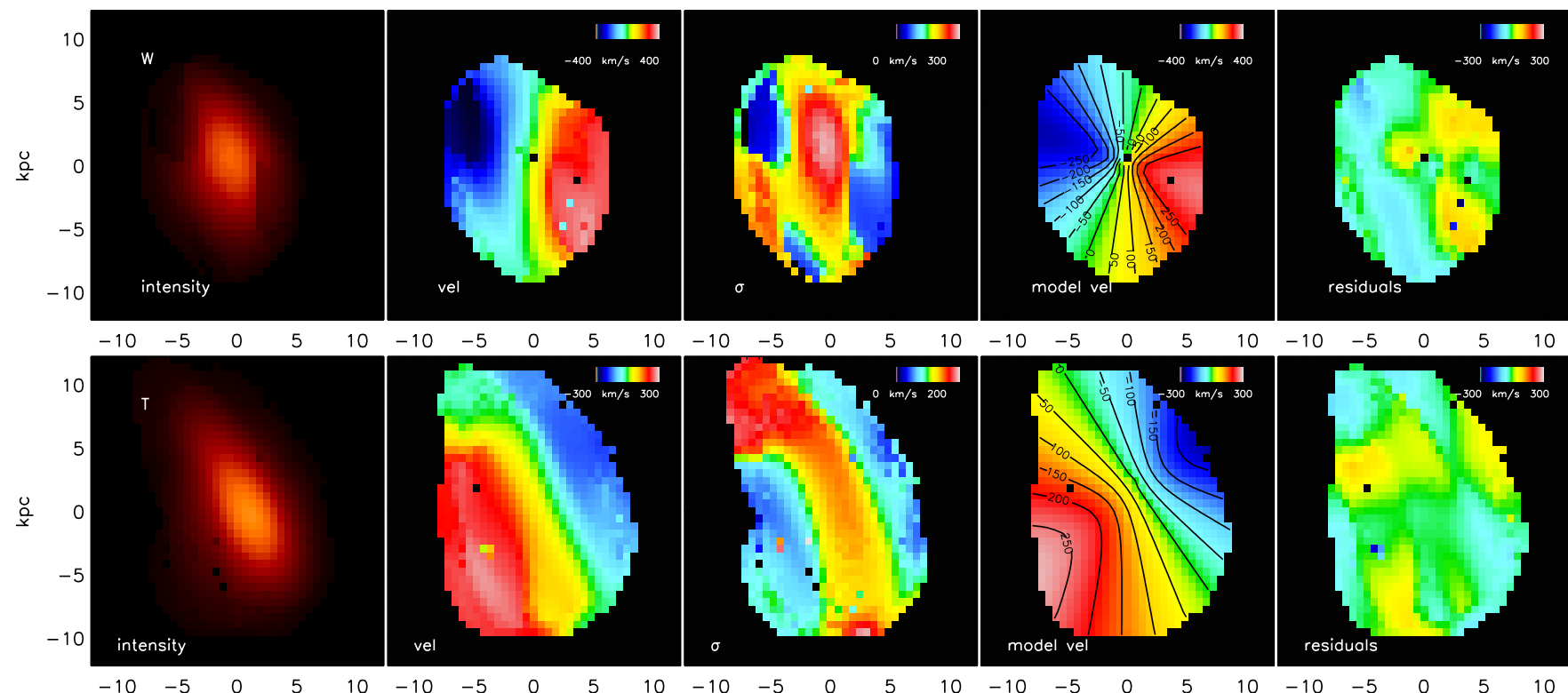

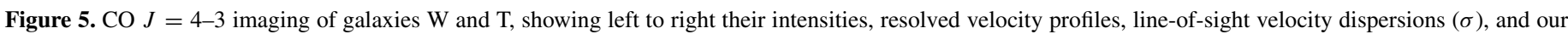

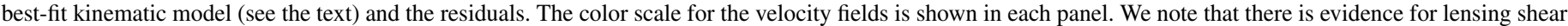
in the velocity field and line-of-sight velocity dispersion for $\mathrm{T}$.

(A color version of this figure is available in the online journal.)

As we saw in Sections 3.6 and 3.7-their star formation is not centrally concentrated as would be the case in local starbursts. Instead, $\mathrm{W}$ and $\mathrm{T}$ are undergoing widespread star formation in disks that are supported partly by rotation, as envisaged for main-sequence galaxies, and share many of the properties of massive star-forming disks (e.g., Förster Schreiber et al. 2009). Some other factor-presumably their interaction-has taken them into the realm of sSFR reserved for starbursts.

For the $Q$ parameter $^{53}$ (Toomre 1964; Goldreich \& LyndenBell 1965), which describes the stability of disks and which has been found to tend toward unity in stable situations (in models with and without feedback; Hopkins et al. 2012), we find values of $\approx 0.35$ for both $\mathrm{W}$ and $\mathrm{T}$, with a plausible range that falls just short of unity. This suggests the disks are unstable and that the gas therein is prone to condense and form stars on a timescale shorter than the rotational period.

\subsection{Gas Properties and $\alpha_{C O}$}

The brightness temperature $\left(T_{\mathrm{b}}\right)$ ratios measured for both $\mathrm{W}$ and $\mathrm{T}$ are higher than expected (Table 2), where we usually see $r_{3-2 / 1-0} \sim 0.52 \pm 0.09$ and $r_{4-3 / 1-0} \sim 0.41 \pm 0.07$ for SMGs (Harris et al. 2010; Ivison et al. 2011; Bothwell et al. 2013), which suggests that the thermalized $\mathrm{CO} J=3-2$ filling factors may be close to unity and that we may have found similar sizes in CO $J=1-0$ and $J=3-2$ if our spatial resolution in the latter had been two times higher.

For a $\mathrm{CO}$ to $\mathrm{H}_{2}+\mathrm{He}$ conversion factor, $\alpha_{\mathrm{CO}}=0.8 M_{\odot}$ $\left(\mathrm{K} \mathrm{km} \mathrm{s}^{-1} \mathrm{pc}^{2}\right)^{-1}$, as commonly adopted for IR-luminous starbursts where the gas is not in virialized individual clouds (Bolatto et al. 2013), the gas masses we determine for W, T, C, and $\mathrm{M}$ are $110,83.7,12.8$, and $17.8 \times 10^{9} M_{\odot}$ (corrected for lensing).

If we were instead to adopt the all-sample average gas/dust mass ratio for nearby galaxies $\left(M_{\text {gas }} / M_{\text {dust }}=91_{-36}^{+60} ;\right.$ Sandstrom

\footnotetext{
53 Usually ascribed to a seminal paper on the stability of stellar disks (Toomre 1964), which contains no mention of $Q$.
}

et al. 2013), we would determine gas masses for $\mathrm{W}$ and $\mathrm{T}$ of 19.0 and $11.5 \times 10^{9} M_{\odot}$, yielding gas/dust-motivated $\alpha_{\mathrm{CO}}$ values of 1.4 and $1.1 M_{\odot}\left(\mathrm{K} \mathrm{km} \mathrm{s}^{-1} \mathrm{pc}^{2}\right)^{-1}$, respectively. Due to the uncertainty in $\kappa_{850}$, etc., we regard these as accurate to a factor of two times at best. For an environment with sub-solar metallicity, for example, $\alpha_{\mathrm{CO}}$ would rise in line with $M_{\text {gas }} / M_{\text {dust }}$.

A dynamically motivated estimate of $\alpha_{\mathrm{CO}}$ can be made by determining the difference between the dynamical and stellar masses in each system, since this represents the total plausible mass of gas. ${ }^{54}$ Via this method, we find $\alpha_{\mathrm{CO}}=0.6$ and $0.3 M_{\odot}\left(\mathrm{K} \mathrm{km} \mathrm{s}^{-1} \mathrm{pc}^{2}\right)^{-1}$ for $\mathrm{W}$ and $\mathrm{T}$, respectively, where the uncertainty, $\pm 0.3 \mathrm{dex}$, is due almost entirely to the notoriously difficult estimate of stellar mass (Section 3.4).

Finally, we can estimate $\alpha_{\mathrm{CO}}$ using its relationship with gasphase metallicity and $\mathrm{CO}$ surface brightness, as developed by Narayanan et al. (2012b), exploiting the resolved imaging in CO $J=1-0$ acquired here. For solar metallicity (Chapman et al. 2005) and the sizes that have been measured accurately in our JVLA imaging (Section 3.6; Table 2) we find $\alpha_{\mathrm{CO}}=0.8$ and $0.7 M_{\odot}\left(\mathrm{K} \mathrm{km} \mathrm{s}^{-1} \mathrm{pc}^{2}\right)^{-1}$ for $\mathrm{W}$ and $\mathrm{T}$, respectively.

Our mean is $\alpha_{\mathrm{CO}}=0.8 \pm 0.4 M_{\odot}\left(\mathrm{K} \mathrm{km} \mathrm{s}^{-1} \mathrm{pc}^{2}\right)^{-1}$, in line with the value usually adopted for starburst galaxies, with a range similar to that reported by Downes \& Solomon (1998), $0.3-1.3 M_{\odot}\left(\mathrm{K} \mathrm{km} \mathrm{s}^{-1} \mathrm{pc}^{2}\right)^{-1}$. To reconcile our dynamical measurement of $\alpha_{\mathrm{CO}}$ with $3.2 M_{\odot}\left(\mathrm{K} \mathrm{km} \mathrm{s}^{-1} \mathrm{pc}^{2}\right)^{-1}$, which Genzel et al. (2010) argue is appropriate for all disks, regardless of redshift, would require a buried AGN to dominate the near-IR light, causing us to overestimate $M_{\text {stars }}$.

Adopting our best estimate for $\alpha_{\mathrm{CO}}$, we find that $\mathrm{W}$ and $\mathrm{T}$ are both approaching the accepted criterion for maximal starbursts, with star formation efficiencies, $L_{\mathrm{IR}} / M_{\text {gas }}$, of 370 and $210 L_{\odot} M_{\odot}^{-1}$. C and $\mathrm{M}$ are higher still, though with considerable uncertainties. The Eddington limit is believed to be around $500 L_{\odot} M_{\odot}^{-1}$; above this level, the radiation pressure should

\footnotetext{
54 Bearing in mind that any AGN contribution to the rest-frame near-IR luminosity will led to an overestimate of stellar mass.
} 
quickly expel the gas via radiation pressure from young, massive stars on dust (Scoville 2004).

Feedback due to the aforementioned radiation pressure will lengthen the total duration of star formation episodes, or lead to a series of short bursts. Ignoring this, the gasconsumption timescale implied for the galaxies comprising HATLAS J084933 is $\tau_{\text {gas }} \sim M_{\text {gas }} /$ SFR $\approx 15-45$ Myr, consistent with an interaction-driven period of enhanced star formation (Scoville et al. 1986), as already hinted by the morphologically tortured state of $\mathrm{C}$.

The stellar masses determined in Section 3.4 for $\mathrm{W}, \mathrm{T}$, and $\mathrm{C}$ suggest gas fractions, $f_{\text {gas }}=M_{\text {gas }} /\left(M_{\text {gas }}+M_{\text {stars }}=\right.$ $36 \%-45 \%$. These gas fractions are the top end of the range that Narayanan et al. (2012a) showed to be difficult to reconcile with cosmological simulations.

\subsection{Relevance for Star-formation Laws}

Data of the quality gathered here have the potential to test whether the rules and prescriptions developed to describe star formation in the local universe (e.g., Schmidt 1959; Kennicutt 1989) can be applied to the interstellar medium of distant, gasrich starbursts like HATLAS J084933. Are the data consistent with a simple, volumetric star formation law in which the SFR is $\sim 1 \%$ of the molecular gas mass per local free-fall time, as argued by Krumholz et al. (2012)? Are their SFRs more closely related to the orbital periods of their entire galactic disks (e.g., Genzel et al. 2010; Daddi et al. 2010), or to the quantity of gas above some density threshold (e.g., Lada et al. 2010; Heiderman et al. 2010)?

We begin this discussion in the upper panel of Figure 6: a luminosity-luminosity plot of the observables, $L_{\mathrm{IR}}$ and $L_{\mathrm{CO}}^{\prime}$, for low- and high-redshift SFGs, e.g., $z=0$ disks, luminous infrared galaxies (LIRGs) and ULIRGs and $z=1-2.5 \mathrm{BzK}$ galaxies, taken from Genzel et al. (2010), and for SMGs. For this plot, we need make no assumption about the appropriate value of $\alpha_{\mathrm{CO}}$. The quality and depth of our continuum and $\mathrm{CO}$ data mean that the errors bars for $\mathrm{W}$ and $\mathrm{T}$ can barely be discerned. Using $L_{\mathrm{CO}}^{\prime}$ measurements made only in $\mathrm{CO} J=1-0$, thereby avoiding the need to adopt a $T_{\mathrm{b}}$ ratio, alongside selfconsistent measurements of $L_{\mathrm{IR}}$, we find no compelling evidence that different relations apply to SMGs and other SFGs, in line with the findings of Ivison et al. (2011). Here, we use the sample of SFGs from Genzel et al. (2010) and find essentially the same result.

It is noteworthy that while component $\mathrm{T}$ contributes most to $I_{\mathrm{CO}}$ or $L_{\mathrm{CO}}^{\prime}$ in all three observed $\mathrm{CO}$ transitions, component $\mathrm{W}$ is significantly brighter in the continuum at IR-through-radio wavelengths. We find a similar variation between $\mathrm{W}$ and $\mathrm{T}$ in relation to the far-IR/radio flux ratio, with $q_{\mathrm{IR}}=2.30 \pm 0.08$ and $2.53 \pm 0.13$, respectively, spanning (and consistent with) the tight correlation for SFGs $\left(q_{\mathrm{IR}} \approx 2.4\right.$, e.g., Yun et al. 2001). One might think their different line-to-continuum ratios would lead to a relatively large difference in their positions on the plot of $L_{\mathrm{IR}}$ versus $L_{\mathrm{CO}}^{\prime}$ (Figure 6, upper panel). In fact, W and T both lie comfortably among the scatter of SMGs, suggesting that this scatter may be intrinsic rather than due to measurement error.

Moving to the standard surface-density Schmidt-Kennicutt $(\mathrm{S}-\mathrm{K})$ relation, $\sum_{\mathrm{SFR}} \propto \sum_{\text {gas }}^{N}$, shown as the middle panel of Figure 6 , it is now necessary to adopt value(s) for $\alpha_{\mathrm{CO}}$ appropriate for SFGs and SMGs. Genzel et al. (2010) argue that $3.2 M_{\odot}\left(\mathrm{K} \mathrm{km} \mathrm{s}^{-1} \mathrm{pc}^{2}\right)^{-1}$ is appropriate for disks, regardless of redshift; our dynamical $\alpha_{\mathrm{CO}}$ constraints preclude the adoption of this value for $\mathrm{W}$ and $\mathrm{T}$. We use 3.2 and $0.8 M_{\odot}\left(\mathrm{K} \mathrm{km} \mathrm{s}^{-1} \mathrm{pc}^{2}\right)^{-1}$
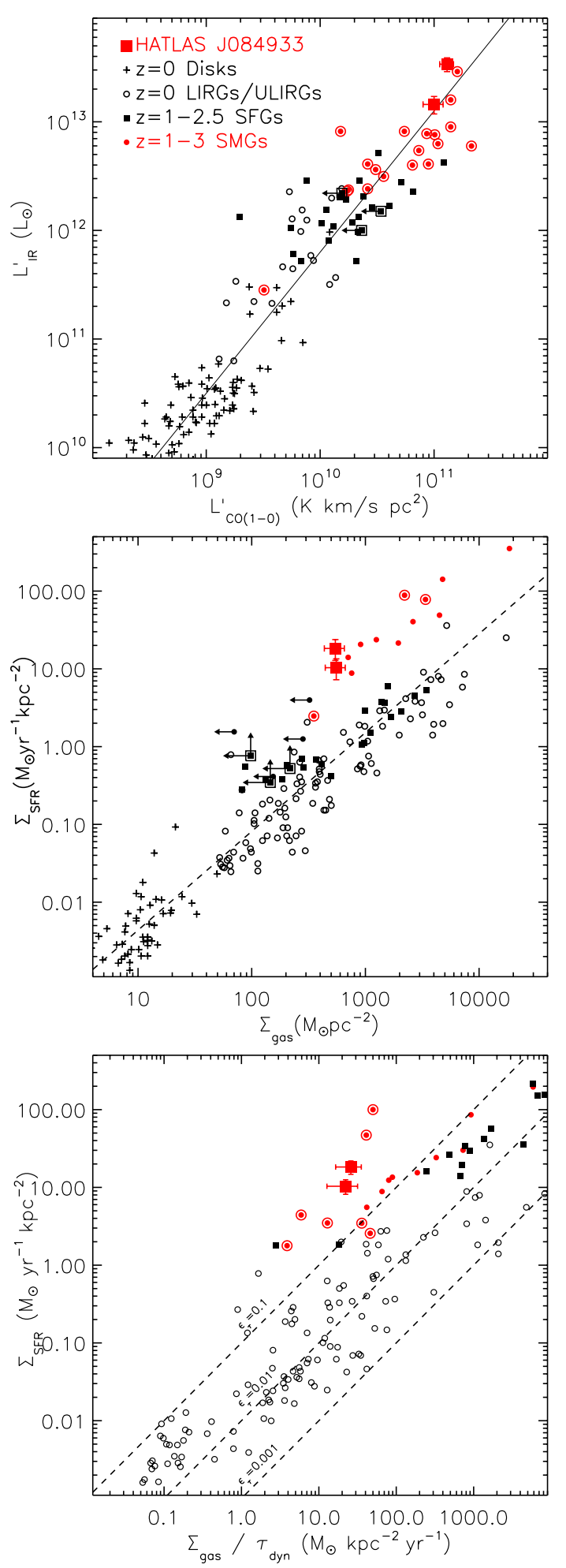

Figure 6. Plots relating the rate or surface density of star formation with the available gas. Components $\mathrm{W}$ and $\mathrm{T}$ are shown as red squares, with error bars, in each plot. Circled red symbols denote high-redshift measurements made directly in CO $J=1-0$. Low- and high-redshift star-forming galaxies (SFGs), e.g., $z=0$ disks, LIRGs and ULIRGs, and $z=1-2.5$ BzK galaxies, are taken from Genzel et al. (2010) for all three plots. Top: $L_{\mathrm{IR}}$ vs. $L_{\mathrm{CO}}^{\prime}$, where we find no compelling evidence that SMGs follow a different relation than the other SFGs. Middle: surface-density S-K relation, where $\mathrm{W}$ and $\mathrm{T}$ lie among hyperluminous SMGs, such as SMM J02399-0136 and GN 20, offset from the relation for other SFGs. Lower: Elmegreen-Silk relation between star formation surface density and the ratio of gas surface density and dynamical timescale. W and T exhibit extreme star formation efficiencies, even relative to SMGs. The fraction of their available gas converted into stars per dynamical timescale, $\epsilon_{\tau}$, is $>0.1$. (A color version of this figure is available in the online journal.) 
for SFGs and SMGs, respectively, acknowledging that this is overly simplistic ${ }^{55}$ (e.g., Narayanan et al. 2012b). We find W and T nestled among the other SMGs again (Figure 6), but this time the SMGs are offset ${ }^{56}$ significantly from the relation for other SFGs.

The lower panel of Figure 6 shows the Elmegreen-Silk (E-S) relation between star formation surface density and the ratio of gas surface density and dynamical timescale. ${ }^{57}$ Taken at face value, $\mathrm{W}$ and $\mathrm{T}$ exhibit extreme star formation efficiencies. Several other SMGs are equally extreme, converting $>10 \%$ of their available gas into stars per dynamical timescale $\left(\epsilon_{\tau}>0.1\right)$. Krumholz et al. (2012) have argued in favor of a simple volumetric star formation law, linking Galactic clouds, SMGs, and all scales between the two, with projection effects leading to the observed scatter. Here, we have discovered two disks exhibiting star formation of such ferocity that they are capable of converting $>10 \%$ of their molecular gas into stars with each rotation. We require a number of parameters to conspire together if we are to escape the conclusion that the gas in these disks does not obey the simple volumetric star formation law proposed by Krumholz et al. (2012).

Before leaving the S-K and E-S laws, we note that although $L_{\mathrm{IR}}$ is an effective tracer of $\sum_{\mathrm{SFR}}$ in Eddington-limited starforming disks, Ballantyne et al. (2013) have argued that velocityintegrated CO line intensity is a poor proxy for $\sum_{\text {gas }}$. Resolved observations of the mid-IR rotational lines of $\mathrm{H}_{2}$ may provide the ultimate probe of the star formation relations in high-redshift disks, requiring a far-IR space interferometer, such as FIRI (Helmich \& Ivison 2008).

\subsection{Future Evolution of the System}

What does the HATLAS J084933 system look like in the present day?

The extreme starbursts that brought this galaxy to our attention via Herschel are likely to have been triggered by one or more interactions (Engel et al. 2010). Certainly, C is distorted, morphologically, and the distances between these galaxies are consistent with periods of intense star formation during merger simulations (e.g., Springel et al. 2005).

However, without knowledge of the dark matter halo they inhabit and the transverse velocities of $\mathrm{W}, \mathrm{T}, \mathrm{C}$, and $\mathrm{M}$, it is impossible to be sure whether these galaxies are gravitationally bound, let alone whether they will merge. We find that the total energy, $1 / 2 v^{2}+\Phi\left(\mathrm{km} \mathrm{s}^{-1}\right)^{2}$, of each of these systems is negative-indicating that the galaxies are bound to one another-if their dynamical masses represent as little as $50 \%$ of the total. This is confirmed by $N$-body simulations, starting from the observed velocities and positions on the sky, and assuming that the transverse velocities do not exceed those in the line of sight. If these galaxies inhabit an expected dark matter halo of mass $\approx 10^{13} M_{\odot}$ (e.g., Amblard et al. 2011; Hildebrandt et al. 2013), it is difficult to escape the conclusion that the system is bound.

\footnotetext{
55 Changing $\alpha_{\mathrm{CO}}$ by a factor two (in opposite directions) for the SFGs and SMGs would bring them in line with one another. Because of the potential for a large systematic error in measurements of stellar mass, among other things, we view such a change as plausible.

56 Any change in the area used to calculate the gas or SFR surface density results in a point shifting parallel to the power-law relation seen in this plot. 57 Although we have estimated the orbital timescale, in this calculation, we use the dynamical timescale of the disk, calculated using the half-light radius and the velocity dispersion, since Krumholz et al. (2012) show that use of the orbital time can artificially increase the apparent star formation efficiency.
}

Taking another approach, we searched the $(500 / h)^{3} \mathrm{Mpc}^{3}$ Millennium Simulation at $z=2$ (Springel et al. 2005). In each friends-of-friends halo we identified all of the sub-halos, as well as the most massive sub-halo, which we take to be akin to $\mathrm{W}$. We then computed the velocity and spatial offset between the most massive sub-halo and all the other sub-halos and tracked the $\approx 200$ cases where there are three sub-halos within a radius of $300 \mathrm{kpc}$ (comoving) with velocities $>90$, $>600$, and $>800 \mathrm{~km} \mathrm{~s}^{-1}$ in projection (i.e., taking into account likely line-of-sight distances and transverse velocities). Those halos, typically $\approx 1.6 \times 10^{13} M_{\odot}$ at $z=2$, end up with a median mass of $\approx 3.8_{-1.9}^{+5.5} \times 10^{14} M_{\odot}$ in the present day $(90 \%$ lower limit, $\left.1.6 \times 10^{14} M_{\odot}\right)$ - structures that are thought to have a space density of a few $\times 10^{-7} \mathrm{Mpc}^{-3}$ (e.g., Reiprich \& Böhringer 2002; Vikhlinin et al. 2009).

The scale over which we see the galaxies comprising HATLAS J084933, $\approx 100 \mathrm{kpc}$, is similar to the core radius of rich clusters of galaxies, and the separation of $\mathrm{W}$ and $\mathrm{T}$ (in terms of $\mathrm{kpc}$ and $\mathrm{km} \mathrm{s}^{-1}$ ) is typical of binary galaxies (e.g., Turner 1976; Schweizer 1987) and within a factor of two times of the projected separation of the two dominant galaxies in the Coma cluster. ${ }^{58}$ We conclude that such systems can survive for a long time and are not necessarily in the process of merging. The four starbursts in HATLAS J084933 may instead be signposting a protocluster, where the majority of members are likely well below the detection thresholds of our panchromatic imaging. It is ironic that deliberate attempts to select clusters at submm wavelengths (targeting radio galaxies and quasars, for example, Stevens et al. 2003; De Breuck et al. 2004; Greve et al. 2007; Priddey et al. 2008) have met with only limited success, while serendipitous discoveries are becoming relatively commonplace (e.g., Riechers et al. 2010).

\subsection{On the Selection of HyLIRGs, and Their Rarity}

Having resolved HATLAS J084933 into its constituent parts, if we place $\mathrm{W}$ and $\mathrm{T}$ individually on the Harris et al. plot of $L_{\mathrm{CO}}^{\prime}$ versus FWHM line width (Figure 7) we find that $\mathrm{W}$ has moved further within the scatter of the intrinsic relation, with a predicted amplification close to unity. The predicted amplification for $\mathrm{T}$, $\mu \approx 2$, is consistent with the lens model presented in Section 3.2.

We can estimate the space density of objects as rare as HATLAS J084933 by utilizing the semiempirical model of Hopkins et al. (2010) and Hayward et al. (2013). We start with halo mass functions and merger rates over the redshift range of interest derived from the Millennium Simulation (Springel et al. 2005), assigning galaxies to each halo following the abundancematching techniques described in Conroy \& Wechsler (2009) to yield the galaxy-galaxy merger rate as a function of mass, merger-mass ratio, and redshift. Utilizing the stellar mass functions of Marchesini et al. (2009), with extrapolations to higher redshifts following Fontana et al. (2006), we calculate the merger rate for all galaxies with a stellar mass ratio equal to or greater than that of $\mathrm{W}$ and $\mathrm{T}$, with one component at least as massive as $\mathrm{W}$. Integrating the merger rates between $z=2$ and $6(t=2.4 \mathrm{Gyr})$, where we are sensitive to such events via $H$-ATLAS, results in a space density of approximately $10^{-7} \mathrm{Mpc}^{-3}$, similar to the space density predicted for extreme HyLIRGs (Lapi et al. 2011; recall also Section 3.11). For the typical gas-consumption timescale found in Section 3.9,

\footnotetext{
58 Coma (A1656) is a B(inary)-type cluster, with two bright, supergiant galaxies, NGC 4874 and NGC 4889, close to each other in the cluster core (Rood \& Sastry 1971).
} 


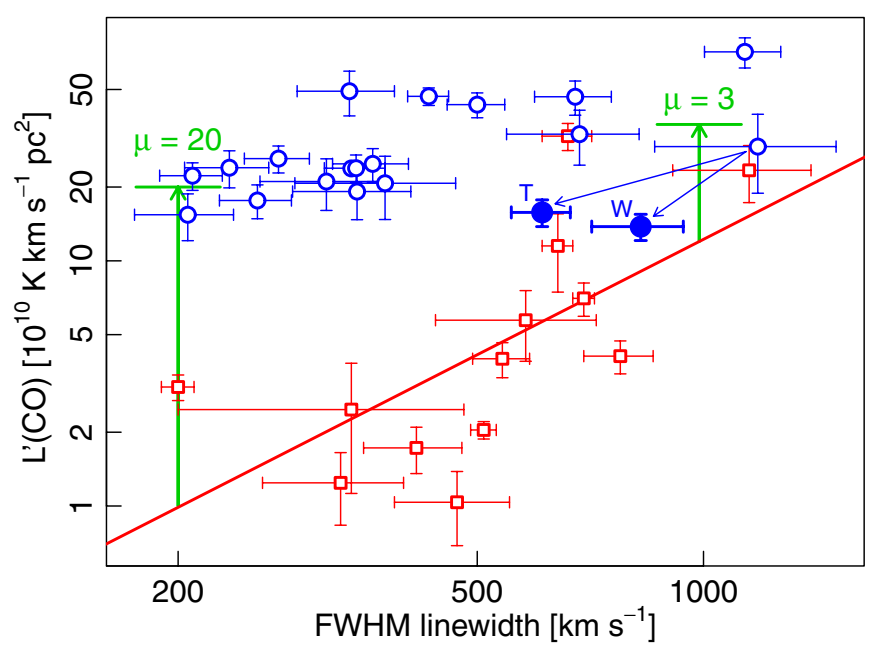

Figure 7. $L_{\mathrm{CO}}^{\prime}$ vs. CO $J=1-0 \mathrm{FWHM}$ line widths for SMGs with redshifts, fluxes, and magnifications from the literature (red squares-for details, see Harris et al. 2012, from whence this figure has been adapted). The power-law fit to these data represents an estimate of the intrinsic line luminosity vs. line width relation for typical SMGs. Blue circles represent the lensed sample from Harris et al. (2012). The point for HATLAS J084933 has been replaced with those appropriate for components $\mathrm{W}$ and $\mathrm{T}$. W has moved well within the scatter of the intrinsic relation, with a predicted amplification consistent with unity. The predicted amplification for $\mathrm{T}$ is consistent with the lens model presented in Section 3.2, $\mu=1.5 \pm 0.2$.

(A color version of this figure is available in the online journal.)

$\tau_{\mathrm{SMG}} \approx 15-45 \mathrm{Myr}$, we then expect to find $\approx 3-9$ such galaxies in the $\approx 100 \mathrm{deg}^{2}$ explored so far in $H$-ATLAS. The vast majority of bright Herschel galaxies have been shown to be lensed (Negrello et al. 2010), but the low end of this range is plausible. This suggests that the most luminous phase of these galaxies has a short duration, $\lesssim 15 \mathrm{Myr}$, and/or that such systems require some other rare property-counter-rotating disks being one possibility. Stellar mass functions at high redshift have significant uncertainties associated with them; nonetheless, this calculation emphasizes the rarity of major mergers, such as that between $\mathrm{W}$ and $\mathrm{T}$, and illustrates the efficiency of the method employed here to find extreme HyLIRGs. The observed surface density of these systems is such that even with short durations for their most luminous phases, they can end up as a subset of the massive present-day clusters discussed in Section 3.11.

HATLAS J084933 was selected on the basis of its broad $\mathrm{CO}$ line profile as a low-magnification, intrinsically luminous system, so it is ironic that its wide line profile is in part due to the multiple nature of the source and that future searches for single maximal-luminosity galaxies may require the selection of less extreme profiles. However, fundamentally we conclude that the method explored here for the selection of the most extreme starburst events in the universe shows promise.

\section{CONCLUSIONS}

1. Exploiting the relationship between CO luminosity and line width determined for unlensed starbursts, we have identified and removed gravitationally lensed sources from the brightest galaxies found in the widest extragalactic Herschel survey to yield a sample of intrinsically luminous galaxies. Here, we report deep panchromatic followup observations of the best candidate HyLIRG system, HATLAS J084933, with subarcsecond spectral and spatial resolution, which have led to the discovery of at least four starbursting galaxies across $\mathrm{a} \approx 100 \mathrm{kpc}$ region at $z=2.41$, each with a significant mass of stars.

2. The two brightest galaxies, $\mathrm{W}$ and $\mathrm{T}$, are separated by $\sim 85 \mathrm{kpc}$ on the sky. W suffers no gravitational amplification. $\mathrm{T}$ is marginally lensed by a foreground lenticular.

3. The panchromatic SEDs of $\mathrm{W}$ and $\mathrm{T}$ reveal that both are associated with high-luminosity events-HyLIRGs. If contributions to $L_{\mathrm{IR}}$ from AGNs are small, and to date we have found no reason to suspect otherwise, then $\mathrm{W}$ and $\mathrm{T}$ have $\log L_{\mathrm{IR}} \sim 13.52$ and $13.16, T_{\text {dust }}=36-40 \mathrm{~K}$, and are generating $\approx 3400$ and $\approx 1500 M_{\odot} \mathrm{yr}^{-1}$ of stars, respectively.

4. Two other gas-rich galaxies, $\mathrm{M}$ and $\mathrm{C}$, detected nearby, make HATLAS J084933 reminiscent of the first SMG found and the best-studied HyLIRG to date, SMM J02399-0136, which comprises a complex galactic nursery with three massive stellar components, each seen during a different evolutionary stage (C. Ferkinhoff et al., in preparation). The four galaxies known to comprise HATLAS J084933 are most likely bound. $N$-body simulations and comparison with the Millennium Simulation suggest that in the present day, the system may resemble a B(inary)-type cluster of mass, $\approx 10^{14.6} M_{\odot}$.

5. Subarcsecond interferometric imaging reveals that the two brightest galaxies span $\sim 3 \mathrm{kpc}$ FWHM in CO $J=4-3$, and in submm and radio continuum, and roughly double that in $\mathrm{CO} J=1-0$. Alongside the detection of [C II], this supports a scenario involving a widespread burst of intense star formation.

6. Exquisite 3D imaging from JVLA and IRAM PdBI reveals counter-rotating gas disks in $\mathrm{W}$ and $\mathrm{T}$, a scenario that has long been predicted to lead to the most intense bursts of star formation. This suggests that similarly luminous galaxies will often be found in pairs. Their modest velocity dispersions mean that the disks are prone to instabilities on many scales, even that of the disks themselves; they are undergoing coherent, extreme starbursts, at close to the Eddington limit, which are not confined to their nuclei. Despite their disk-like morphologies and dynamics, their sSFRs place them $\sim 5$ times above the main sequence.

7. $\mathrm{W}$ and $\mathrm{T}$ have $\mathrm{CO}$ line intensities and widths typical of the brightest SMGs, with slightly higher $T_{\mathrm{b}}$ ratios. Three independent estimates of the CO-to- $\mathrm{H}_{2}$ conversion factor-exploiting the dynamical mass, the mass of dust, and a recent metallicity-dependent relation-suggest $\alpha_{\mathrm{CO}}=0.8 \pm 0.4 M_{\odot}\left(\mathrm{K} \mathrm{km} \mathrm{s}^{-1} \mathrm{pc}^{2}\right)^{-1}$ and hence gas masses in $\mathrm{W}$ and $\mathrm{T}$ of $\approx 10^{11} M_{\odot}$. Their gas fractions, $\sim 40 \%$, are difficult to reconcile with cosmological simulations.

8. We have determined that the single-dish $\mathrm{CO}$ line profile of HATLAS J084933 is exceptionally broad because W and $\mathrm{T}$ have relatively wide lines, broadened further in the Harris et al. (2012) integrated GBT spectrum by the modest velocity offset between them, and by contributions from a number of faint $\mathrm{CO}$ clumps slightly redward of the two dominant components. We can thus reconcile the GBT CO line profile with that of the various galaxies found to comprise HATLAS J084933. These situations likely happen only rarely, even in the widest extragalactic Herschel surveys. We estimate that only a few systems of this nature will be found in every $100 \mathrm{deg}^{2}$ surveyed.

9. Placing $\mathrm{W}$ and $\mathrm{T}$ individually on the Harris et al. plot of $L_{\mathrm{CO}}^{\prime}$ versus FWHM CO line width, $\mathrm{W}$ and $\mathrm{T}$ move further within the scatter of the intrinsic relation, with a predicted 
amplification close to unity. We conclude that the method outlined here for the selection of extreme starbursts can indeed separate HyLIRGs from the more numerous, less luminous, strongly lensed population of IR-bright galaxies, and may also pinpoint some distant clusters of starbursting protoellipticals.

10. Plotting $L_{\mathrm{IR}}$ versus $L_{\mathrm{CO}}^{\prime}, \mathrm{W}$ and $\mathrm{T}$ lie among the SMGs on a single relation that is consistent with all SFGs. They also lie among the SMGs on the classic S-K and E-S plots, but the SMGs now follow a relation that is distinct from those of other SFGs. Their star formation efficiency is significantly higher, and is difficult to reconcile with a simple volumetric star formation law in which $\sim 1 \%$ of the available gas is converted into stars during each local free-fall time.

Understanding systems as luminous as HATLAS J084933 represents a challenge. However, the observations presented here have shed considerable light on the characteristics that likely lead to the formation-however briefly-of the most luminous SFGs in the universe. Observations scheduled for Cycle 1 with the Atacama Large Millimeter/Submillimeter Array promise to reveal more about this dramatic and complex protogalactic environment, probing the structure of the disks and sensitive to the presence and influence of buried AGNs.

R.J.I. acknowledges support from the European Research Council (ERC) in the form of Advanced Grant, CosmicisM. The Herschel-ATLAS is a project with Herschel, which is an ESA Space Observatory with science instruments provided by European-led Principal Investigator consortia and with important participation from NASA. The $H$-ATLAS Web site is www.h-atlas.org. US participants in $H$-ATLAS acknowledge support from NASA through a contract from JPL. I.R.S. acknowledges support from STFC and ERC. M.N. and G.dZ. acknowledge financial support from ASI/INAF Agreement I/072/09/0.

Facilities: CARMA, CSO, Herschel, HST, IRAM: Interferometer, VLA, Keck:II, SMA, Spitzer, ESO: VISTA

\section{REFERENCES}

Alexander, D. M., Brandt, W. N., Smail, I., et al. 2008, AJ, 135, 1968 Amblard, A., Cooray, A., Serra, P., et al. 2011, Natur, 470, 510

Aravena, M., Younger, J. D., Fazio, G. G., et al. 2010, ApJL, 719, L15 Ballantyne, D. R., Armour, J. N., \& Indergaard, J. 2013, ApJ, 765, 138 Bekki, K. 2001, ApJ, 546, 189

Bolatto, A. D., Wolfire, M., \& Leroy, A. K. 2013, ARA\&A, in press (arXiv:1301.3498)

Borne, K. D., Bushouse, H., Lucas, R. A., \& Colina, L. 2000, ApJL, 529, L77 Bothwell, M. S., Smail, I., Chapman, S. C., et al. 2013, MNRAS, 429, 3047

Bruzual, G., \& Charlot, S. 2003, MNRAS, 344, 1000

Chabrier, G. 2003, PASP, 115, 763

Chapman, S. C., Blain, A., Smail, I., \& Ivison, R. 2005, ApJ, 622, 772

Conroy, C., \& Wechsler, R. H. 2009, ApJ, 696, 620

Courteau, S. 1997, AJ, 114, 2402

da Cunha, E., Charlot, S., \& Elbaz, D. 2008, MNRAS, 388, 1595

Daddi, E., Elbaz, D., Walter, F., et al. 2010, ApJL, 714, L118

De Breuck, C., Bertoldi, F., Carilli, C., et al. 2004, A\&A, 424, 1

Di Matteo, P., Combes, F., Melchior, A.-L., \& Semelin, B. 2007, A\&A, 468, 61

Dowell, C. D., Allen, C. A., Babu, R. S., et al. 2003, Proc. SPIE, 4855, 73

Downes, D., \& Solomon, P. M. 1998, ApJ, 507, 615

Dunne, L., Eales, S., Edmunds, M., et al. 2000, MNRAS, 315, 115

Dunne, L., \& Eales, S. A. 2001, MNRAS, 327, 697

Dwek, E., Staguhn, J. G., Arendt, R. G., et al. 2011, ApJ, 738, 36

Eales, S., Dunne, L., Clements, D., et al. 2010, PASP, 122, 499

Elbaz, D., Dickinson, M., Hwang, H. S., et al. 2011, A\&A, 533, A119

Emerson, J. P., \& Sutherland, W. J. 2010, Proc. SPIE, 7733, 4
Engel, H., Tacconi, L. J., Davies, R. I., et al. 2010, ApJ, 724, 233

Fazio, G. G., Hora, J. L., Allen, L. E., et al. 2004, ApJS, 154, 10 Fontana, A., Salimbeni, S., Grazian, A., et al. 2006, A\&A, 459, 745

Förster Schreiber, N. M., Genzel, R., Bouché, N., et al. 2009, ApJ, 706, 1364

Frayer, D. T., Harris, A. I., Baker, A. J., et al. 2011, ApJL, 726, L22

Gear, W. K., Lilly, S. J., Stevens, J. A., et al. 2000, MNRAS, 316, L51

Genzel, R., Tacconi, L. J., Gracia-Carpio, J., et al. 2010, MNRAS, 407, 2091

Goldreich, P., \& Lynden-Bell, D. 1965, MNRAS, 130, 97

Greve, T. R., Stern, D., Ivison, R. J., et al. 2007, MNRAS, 382, 48

Griffin, M. J., Abergel, A., Abreu, A., et al. 2010, A\&A, 518, L3

Hainline, L. J., Blain, A. W., Smail, I., et al. 2011, ApJ, 740, 96

Harris, A. I., Baker, A. J., Frayer, D. T., et al. 2012, ApJ, 752, 152

Harris, A. I., Baker, A. J., Zonak, S. G., et al. 2010, ApJ, 723, 1139

Hayward, C. C., Kereš, D., Jonsson, P., et al. 2011, ApJ, 743, 159

Hayward, C. C., Narayanan, D., Kereš, D., et al. 2013, MNRAS, 428, 2529

Heiderman, A., Evans, N. J., II, Allen, L. E., Huard, T., \& Heyer, M. 2010, ApJ, 723, 1019

Helmich, F. P., \& Ivison, R. J. 2008, ExA, 23, 245

Helou, G., Soifer, B. T., \& Rowan-Robinson, M. 1985, ApJL, 298, L7

Hildebrandt, H., van Waerbeke, L., Scott, D., et al. 2013, MNRAS, 329, 3230

Hopkins, P. F., Quataert, E., \& Murray, N. 2012, MNRAS, 421, 3488

Hopkins, P. F., Younger, J. D., Hayward, C. C., Narayanan, D., \& Hernquist, L. 2010, MNRAS, 402, 1693

Ibar, E., Ivison, R. J., Best, P. N., et al. 2010a, MNRAS, 401, L53

Ibar, E., Ivison, R. J., Cava, A., et al. 2010b, MNRAS, 409, 38

Ilbert, O., McCracken, H. J., Le Fevre, O., et al. 2013, arXiv:1301.3157

Ilbert, O., Salvato, M., Le Floc'h, E., et al. 2010, ApJ, 709, 644

Iono, D., Peck, A. B., Pope, A., et al. 2006, ApJL, 640, L1

Ivison, R. J., Papadopoulos, P. P., Smail, I., et al. 2011, MNRAS, 412, 1913

Kennicutt, R. C., Jr. 1989, ApJ, 344, 685

Kennicutt, R. C., Jr. 1998, ApJ, 498, 541

Klaas, U., Nielbock, M., Haas, M., Krause, O., \& Schreiber, J. 2010, A\&A, 518, L44

Kovács, A. 2008, Proc. SPIE, 7020, 45

Kovács, A., Omont, A., Beelen, A., et al. 2010, ApJ, 717, 29

Krumholz, M. R., Dekel, A., \& McKee, C. F. 2012, ApJ, 745, 69

Lada, C. J., Lombardi, M., \& Alves, J. F. 2010, ApJ, 724, 687

Lapi, A., González-Nuevo, J., Fan, L., et al. 2011, ApJ, 742, 24

Leong, M., Peng, R., Houde, M., et al. 2006, Proc. SPIE, 6275, 21

Lutz, D., Dunlop, J. S., Almaini, O., et al. 2001, A\&A, 378, 70

Magnelli, B., Lutz, D., Santini, P., et al. 2012, A\&A, 539, A155

Makovoz, D., \& Marleau, F. R. 2005, PASP, 117, 1113

Marchesini, D., van Dokkum, P. G., Förster Schreiber, N. M., et al. 2009, ApJ, 701,1765

Michalowski, M. J., Dunlop, J. S., Cirasuolo, M., et al. 2012, A\&A, 541, A85

Mihos, J. C., \& Hernquist, L. 1994, ApJL, 431, L9

Mihos, J. C., \& Hernquist, L. 1996, ApJ, 464, 641

Morton, D. C., \& Noreau, L. 1994, ApJS, 95, 301

Narayanan, D., Bothwell, M., \& Davé, R. 2012a, MNRAS, 426, 1178

Narayanan, D., Krumholz, M. R., Ostriker, E. C., \& Hernquist, L. 2012b, MNRAS, 421, 3127

Negrello, M., González-Nuevo, J., Magliocchetti, M., et al. 2005, MNRAS, 358,869

Negrello, M., Hopwood, R., De Zotti, G., et al. 2010, Sci, 330, 800

Owen, F. N., \& Morrison, G. E. 2008, AJ, 136, 1889

Pascale, E., Auld, R., Dariush, A., et al. 2011, MNRAS, 415, 911

Pforr, J., Maraston, C., \& Tonini, C. 2012, MNRAS, 422, 3285

Pilbratt, G. L., Riedinger, J. R., Passvogel, T., et al. 2010, A\&A, 518, L1

Poglitsch, A., Waelkens, C., Geis, N., et al. 2010, A\&A, 518, L2

Pope, A., Borys, C., Scott, D., et al. 2005, MNRAS, 358, 149

Priddey, R. S., Ivison, R. J., \& Isaak, K. G. 2008, MNRAS, 383, 289

Reiprich, T. H., \& Böhringer, H. 2002, ApJ, 567, 716

Riechers, D. A., Capak, P. L., Carilli, C. L., et al. 2010, ApJL, 720, L131

Riechers, D. A., Hodge, J., Walter, F., Carilli, C. L., \& Bertoldi, F. 2011, ApJL, 739, L31

Rigby, E. E., Maddox, S. J., Dunne, L., et al. 2011, MNRAS, 415, 2336

Rood, H. J., \& Sastry, G. N. 1971, PASP, 83, 313

Rowlands, K., Dunne, L., Maddox, S., et al. 2012, MNRAS, 419, 2545

Salomé, P., Guélin, M., Downes, D., et al. 2012, A\&A, 545, A57

Sandstrom, K. M., Leroy, A. K., Walter, F., et al. 2013, ApJ (arXiv:1212.1208)

Schmidt, M. 1959, ApJ, 129, 243

Schweizer, L. Y. 1987, ApJS, 64, 411

Scoville, N. 2004, in ASP Conf. Ser. 320, The Neutral ISM in Starburst Galaxies, ed. S. Aalto, S. Huttemeister, \& A. Pedlar (San Francisco, CA: ASP), 253

Scoville, N. Z., Sanders, D. B., \& Clemens, D. P. 1986, ApJL, 310, L77

Smolčić, V., Aravena, M., Navarrete, F., et al. 2012a, A\&A, 548, A4 
Smolčić, V., Navarrete, F., Aravena, M., et al. 2012b, ApJS, 200, 10 Springel, V., White, S. D. M., Jenkins, A., et al. 2005, Natur, 435, 629 Stacey, G. J., Hailey-Dunsheath, S., Ferkinhoff, C., et al. 2010, ApJ, 724, 957

Stevens, J. A., Ivison, R. J., Dunlop, J. S., et al. 2003, Natur, 425, 264

Swinbank, A. M., Smail, I., Longmore, S., et al. 2010, Natur, 464, 733

Taniguchi, Y., \& Shioya, Y. 1998, ApJL, 501, L167

Toomre, A. 1964, ApJ, 139, 1217

Turner, E. L. 1976, ApJ, 208, 20

Vikhlinin, A., Kravtsov, A. V., Burenin, R. A., et al. 2009, ApJ, 692, 1060
Wang, W.-H., Cowie, L. L., van Saders, J., Barger, A. J., \& Williams, J. P. 2007, ApJL, 670, L89

Werner, M. W., Roellig, T. L., Low, F. J., et al. 2004, ApJS, 154, 1 Whitmore, B. C., \& Schweizer, F. 1995, AJ, 109, 960

Wilson, C. D., Petitpas, G. R., Iono, D., et al. 2008, ApJS, 178, 189

Younger, J. D., Fazio, G. G., Huang, J.-S., et al. 2007, ApJ, 671, 1531

Younger, J. D., Fazio, G. G., Wilner, D. J., et al. 2008, ApJ, 688, 59

Younger, J. D., Fazio, G. G., Huang, J.-S., et al. 2009, ApJ, 704, 803

Yun, M. S., Reddy, N. A., \& Condon, J. J. 2001, ApJ, 554, 803 